1

2

3

4

5

8

11

42

43

44

45

46

47

48

\title{
Automated in vivo tracking of cortical oligodendrocytes
}

\author{
${ }^{1}$ The Solomon Snyder Department of Neuroscience, Johns Hopkins University, Baltimore, \\ Maryland, USA \\ ${ }^{2}$ Kavli Neuroscience Discovery Institute \\ ${ }^{3}$ Department of Biomedical Engineering, Johns Hopkins University, Baltimore, Maryland, USA
}

Abstract: 217

Introduction: 644

Materials and Methods: 2054

Results: 2799

Discussion: 1403

8 Figures

3 Supplemental Figures

4 Videos

Corresponding Author:

Dwight E. Bergles 


\section{ABSTRACT}

Oligodendrocytes exert a profound influence on neural circuits by accelerating axon potential conduction, altering excitability and providing metabolic support. As oligodendrogenesis continues in the adult brain and is essential for myelin repair, uncovering the factors that control their dynamics is necessary to understand the consequences of adaptive myelination and develop new strategies to enhance remyelination in diseases such as multiple sclerosis. Unfortunately, few methods exist for analysis of oligodendrocyte dynamics, and even fewer are suitable for in vivo investigation. Here, we describe the development of a fully automated cell tracking pipeline using convolutional neural networks (Oligo-Track) that provides rapid volumetric segmentation and tracking of thousands of cells over weeks in vivo. This system reliably replicated human analysis, outperformed traditional analytic approaches, and extracted injury and repair dynamics at multiple cortical depths, establishing that oligodendrogenesis after cuprizone-mediated demyelination is suppressed in deeper cortical layers. Volumetric data provided by this analysis revealed that oligodendrocyte soma size progressively decreases after their generation, and declines further prior to death, providing a means to predict cell age and eventual cell death from individual time points. This new CNN-based analysis pipeline offers a rapid, robust method to quantitatively analyze oligodendrocyte dynamics in vivo, which will aid in understanding how changes in these myelinating cells influence circuit function and recovery

67 from injury and disease. 


\section{INTRODUCTION}

Advances in genetically encoded fluorescent indicators, CRISPR-mediated gene editing and multiphoton microscopy provide unprecedented opportunities for studying cellular dynamics at single-cell resolution in the brains of living animals. While these approaches hold the potential for profound discoveries about brain function, they also come with a host of quantitative challenges. In particular, living brain tissue is unstable; tissue warping disrupts image quality and uneven refractive indices increase noise and produce anisotropic distortions during longitudinal image acquisition (Lecoq et al., 2019). Moreover, large multi-dimensional datasets are cumbersome to quantify, and often require specialized software for 4D visualization and manual curation (Pidhorskyi et al., 2018). As imaging tools become more advanced and enable researchers to delve deeper into the brain in vivo (Horton et al., 2013), the challenges associated with quantification of enormous datasets become more acute. Further advances depend critically on the availability of robust analysis platforms to rapidly extract multidimensional observations about cellular dynamics.

Developing rigorous analysis tools for in vivo investigation of oligodendrocytes is particularly important. Oligodendrocytes enhance the speed of action potential conduction by ensheathing neuronal axons with concentric wraps of membrane, support neuronal metabolism and control neuronal excitability (Simons and Nave, 2016; Larson et al., 2018). While the population of neurons in the brain remains relatively stable throughout life (Bhardwaj et al., 2006; Ming and Song, 2011), new oligodendrocytes are generated in the adult CNS, allowing for dynamic alteration of myelin patterns in both healthy and pathological conditions (El Waly et al., 2014). This dynamism highlights the need for automated, longitudinal tracking tools to quantify the location, timing and extent of myelin plasticity within defined circuits in response to particular behavioral paradigms, as well as the regeneration of oligodendrocytes after demyelination (Bergles and Richardson, 2015). In this study, we sought to develop fully automated 
methodologies to overcome the analytic challenges associated with longitudinal tracking of oligodendrocytes in vivo. are not readily adaptable to in vivo conditions (Van Valen et al., 2016; Zhong et al., 2016; Nketia et al., 2017; Lugagne et al., 2020; Wang et al., 2020). The few in vivo tracking algorithms that exist are modality specific and cannot be readily adapted to our fluorescent longitudinal datasets

104 (Acton et al., 2002; Nguyen et al., 2011). The closest in vivo tools that can be applied to oligodendrocyte datasets are those developed for analyzing calcium imaging (Pachitariu et al., 2017; Giovannucci et al., 2019). However, calcium imaging tools normally work best with highframe rate videos taken over seconds, rather than image volumes collected on a weekly basis that often experience large-scale tissue warping between imaging sessions. To resolve this longitudinal volumetric tracking challenge, we opted to use convolutional neural networks $(\mathrm{CNN})$, which are known to find accurate efficient solutions to high-dimensional problems.

111 Convolutional kernels allow CNNs to adaptively assess local features and global spatial

112 relationships to make tracking decisions that are more perceptual, or human-like. Moreover, 113 additional techniques such as transfer learning can help trained models generalize to entirely 114 new imaging challenges with minimal new training data (Zhuang et al., 2020), extending their 115 use to other contexts.

Here, we describe the development of Oligo-Track, a fast and reliable cell tracker for in

117 vivo semantic segmentation of oligodendrocyte dynamics across cortical layers in longitudinal

118 imaging experiments. We validated our algorithm using the cuprizone model of demyelination in 119 vivo and show that Oligo-Track outperforms traditional analytic approaches in extracting 120 dynamics of oligodendrogenesis at greater depths than previously available with manual 121 annotation. Moreover, this approach generated volumetric segmentations of tracked cells that 122 were inaccessible to human analysis, due to the considerable time investment required for 123 manual volumetric tracing. This volumetric data revealed that oligodendrocyte soma size varies 
bioRxiv preprint doi: https://doi.org/10.1101/2021.02.12.430879; this version posted February 14, 2021. The copyright holder for this preprint

(which was not certified by peer review) is the author/funder, who has granted bioRxiv a license to display the preprint in perpetuity. It is made available under aCC-BY-NC-ND 4.0 International license.

124 predictably with age and proximity to death, allowing additional information about the timing of 125 oligodendrogenesis and cell death to be extracted from fixed timepoint imaging experiments. 


\section{MATERIALS and METHODS:}

127 Animal care and use

128 Female and male adult mice were used for experiments and randomly assigned to experimental

129 groups. All mice were healthy and did not display any overt behavioral phenotypes, and no

130 animals were excluded from the analysis. Generation and genotyping of BAC transgenic lines

131 from Mobp-EGFP (GENSAT) have been previously described (Hughes et al., 2018). Mice were maintained on a $12 \mathrm{hr}$ light/dark cycle, housed in groups no larger than 5, and food and water were provided ad libitum (except during cuprizone-administration, see below). All animal experiments were performed in strict accordance with protocols approved by the Animal Care and Use Committee at Johns Hopkins University.

\section{Cranial windows}

138 Cranial windows were prepared as previously described (Holtmaat et al., 2012; Hughes et al., 2018; Orthmann-Murphy et al., 2020). Mice aged 7 to 10 weeks were deeply anesthetized with isoflurane ( $5 \%$ with $1 \mathrm{~L} / \mathrm{min} \mathrm{O}_{2}$ induction; $1.5-2 \%$ with $0.5 \mathrm{~L} /$ min maintenance), the head

141 shaved, and the scalp removed to expose the skull. The skull was cleaned and dried and a

142 position over somatosensory cortex (-1.5 $\mathrm{mm}$ posterior and $3.5 \mathrm{~mm}$ lateral from bregma) was

143 marked for drilling. A custom aluminum headplate with a central hole was cemented onto the

144 skull ( $C$ and B Metabond) and fixed in place with custom clamping headbars. A $2 \mathrm{~mm} \times 2 \mathrm{~mm}$

145 square or $3 \mathrm{~mm} \times 3 \mathrm{~mm}$ circle of skull was removed using a high-speed dental drill. A coverslip

146 (VWR, No. 1) the size of the craniotomy was put in its place and sealed with cyanoacrylate glue

147 (Vetbond and Krazy glue).

\section{In vivo two photon microscopy}

150 In vivo imaging was performed as previously described (Orthmann-Murphy et al., 2020). After

151 two to three weeks of recovery from cranial window surgery, baseline images of the cortex were 
152

153

154

acquired with two photon microscopy on a Zeiss LSM 710 microscope (average power at sample $<30 \mathrm{~mW}$ ). Image stacks were $425 \mu \mathrm{m} \times 425 \mu \mathrm{m} \times 550 \mu \mathrm{m}$ or $850 \mu \mathrm{m} \times 850 \mu \mathrm{m} \times 550$ $\mu \mathrm{m}(1024 \times 1024$ pixels; corresponding to layers I - IV), relative to the pia. Mice were subsequently imaged weekly for up to 12 weeks.

\section{Cuprizone treatment}

Directly following baseline two photon image acquisition, mice were switched from regular diet to a diet consisting of milled, irradiated $18 \%$ protein chow (Teklad Global) supplemented with $0.2 \% \mathrm{w} / \mathrm{w}$ bis(cyclohexanone) oxaldihydrazone ("cuprizone," Sigma). Control mice received only the milled chow. After three weeks, mice returned to regular pellet diet for the duration of the recovery period (Orthmann-Murphy et al., 2020).

\section{Analytic pipeline overview}

Timeseries acquired from our two-photon imaging setup were first registered using ImageJ's correct 3D drift plugin (Schindelin et al., 2012; Parslow et al., 2014), which accounted for major alignment shifts from week to week. Registered timeseries were then analyzed crop-by-crop using our segmentation CNN (Seg-CNN) which identified cell somas on a voxel-wise basis. These cell somas were then extracted as individual seeds for our tracking CNN (Track-CNN) that identified the location of each seeded cell soma on a subsequent time point. In parallel, we also developed a cell tracking method based on traditional imaging informatics approaches that used the structural similarity index (SSIM) (Zhou Wang et al., 2004) and local tissue movement calculations to track cells. This heuristic model was used as a baseline to assess the improvements of our Track-CNN approach. Cells tracked by either Track-CNN or our heuristic method were also curated by human researchers using syGlass virtual reality software (Pidhorskyi et al., 2018) to assess the accuracy of tracking. Some of these curated traces were 
177 also returned to the training pipeline to improve our deep learning approaches in a positive-

178 feedback loop (Figure 2A).

179

\section{Training data generation}

All training data was curated by a human expert using syGlass software to provide point coordinates. To obtain volumetric segmentations, we trained an ilastik random forest regressor (Berg et al., 2019) to procure an over-sensitive voxel-wise segmentation model. Then, we excluded every ilastik identified object that did not overlap with a ground truth point coordinate to eliminate false positives in our over-sensitive ilastik model. Datasets were pooled from 12 animals and multiple treatment conditions. Image scales were standardized to $0.83 \mu \mathrm{m} / \mathrm{pixel}$ in $X Y$ and $3 \mu \mathrm{m} /$ pixel in $Z$. Data was cropped to the appropriate input size for each respective neural network: Track-CNN $256 \times 256 \times 64$ voxels, and seg-CNN $128 \times 128 \times 32$ voxels. Overall, Seg-CNN was trained with 6,828 training volumes and 759 validation volumes. TrackCNN was trained with 38,696 volumes and a validation set containing 4,300 volumes.

\section{Segmentation CNN training and inference}

Seg-CNN employed a UNet architecture (Ronneberger et al., 2015) with 3D convolutional kernels built in Pytorch 1.6 (Paszke et al., 2017). The neural network took as input a $256 \times 256$ $\times 64$ voxel volume containing fluorescently labelled oligodendrocytes in a single image channel (Figure 2B). The downsampling branch of the CNN contained 5 convolutional blocks with $5 \times 5$ $\times 5$ filters, batch normalization, and max pooling to downsample the data and extract local features. The upsampling branch employed the same blocks in reverse. Max pooling operations were replaced by trilinear upsampling and $1 \times 1 \times 1$ convolutions to resize the image back to the same input size while extracting global spatial features (Supplementary Figure S1). A final $1 \times 1$ $\times 1$ convolution reduced the output to a two-channel volume which was softmaxed with a threshold of 0.5 to two classes corresponding to background and cell soma. Training was 
performed using a batch size of 2 for 30 epochs on an RTX 2080 Ti GPU. Loss was calculated as cross entropy and optimized using an Adam optimizer with weight decay (Loshchilov and Hutter, 2019) set at a learning rate of $10^{-5}$. During inference on unseen data, entire timeseries were fed to the neural network one timepoint at a time. Our algorithm then acquired $256 \times 256 \times$ 64 voxel crops from these volumes with $50 \%$ overlap to ensure all regions were assessed. Each crop was fed to Seg-CNN individually. The output segmentations of individual crops, with 50\% overlap, were summed together and binarized before being stitched back into a full volume. The final analyzed timeseries is saved and returned to the user (Figure 2B).

\section{Track-CNN training and inference}

Track-CNN employed a similar architecture to Seg-CNN except for a filter size of $7 \times 7 \times 7$ for each convolution and a three channel $128 \times 128 \times 32$ voxel input for our "seed-based" training approach. Seed-based training was employed to draw the attention of our CNN to individual cells in a volume by marking a cell of interest with a binary mask, or "seed" (Figure 3A). The input is thus a three-channel volume where channel 1 contains a raw fluorescence volume cropped from timepoint $t$ and centered around a cell soma of interest. Channel 2 contains the binary mask/seed to indicate the cell of interest on timepoint $t$. All adjacent cells excluding the seed are set to a lower value. Finally, channel 3 contains a raw fluorescence volume cropped from timepoint $t+1$ but centered around the same position as in channel 1 (Figure 3A). In summary, this input provides the raw fluorescence from two consecutive timepoints and also indicates which cell we wish to track from timepoint $t$ to timepoint $t+1$ using the binary mask in channel 2. Thus, the ground truth for optimization is a binary volumetric mask indicating the location of the cell of interest on timepoint $t+1$ (Figure 3A). Training was performed using a batch size of 4 for 18 epochs on an RTX 2080 Ti GPU. Loss was calculated as cross entropy and optimized using Adam optimizer with weight decay (Loshchilov and Hutter, 2019) set at a learning rate of $10^{-5}$ that was dropped to $10^{-6}$ at 13 epochs. During inference, volumes were 
229

230

231

232

cropped around each cell of interest in timepoint $t$ along with seed masks and crops from timepoint $t+1$ to form a three-channel input for Track-CNN. This is repeated until all cells on timepoint $t$ are assessed. Unassociated cells on $t+1$ are then added as newly formed oligodendrocytes to our list of candidate cells, and the analysis continues until all consecutive timepoints are tested (Figure 3A).

\section{Post-processing}

To prevent misalignment of tracks, we included one major post-processing step in our analytic pipeline. We first noticed that, given a human tracked dataset, we could predict the location of a cell body on a subsequent timepoint within $\sim 10$ pixels error by using the local directional vector of the tracks of five nearest neighbor cells from timepoint $t$ to $t+1$ (Figure 3B, C). Thus, given that Track-CNN accurately tracks the majority of cells between consecutive timepoints, we can use the average local vector shift of the five nearest neighbors of any cell to correct for tracks that have severely gone off-target ( > 12 pixel difference from predicted directional vector endpoint). These gross errors can then be re-evaluated. If an unassociated cell exists at the location of the predicted vector endpoint on $t+1$, then the wrongly associated track now points to this unassociated cell. Otherwise, the track is terminated. We also included minor postprocessing steps comprising of: (1) a minimum size threshold of 100 voxels for objects to be considered a cell soma; (2) objects that only exist on a single frame (excluding the first and last frame) are dropped, as they were likely to be debris.

\section{Heuristic baseline method}

Since no baseline methods exist for comparison, we developed an approach to assess the extent to which deep learning outperforms traditional imaging informatics methods. We developed a tracking program in MATLAB R2020a (Mathworks) where cells are cropped from timepoint $t$ and assessed on a pair-wise basis to identify whether its' nearest neighbors on $t+1$ 
correspond to the same cell at timepoint $t$. To determine this association, we employed a few simple heuristics and rules: (1) successful tracking required a structural similarity index (SSIM) greater than 0.2 between cropped volumes from different timepoints. SSIM is an indicator of similarity that considers structure, intensity, and contrast-based differences between images.

We applied the assumption that if a cell exists at $t+1$, the overall local environment should look rather similar at timepoint $t$, thus a correct association would have a moderate to high SSIM. (2) Similar to the post-processing used for Track-CNN, we estimated the average vector of all nearest neighbors to model local tissue movement in a cropped field of view from $t$ to $t+1$. This allowed us to evaluate if the current track from $t$ to $t+1$ flows in the same direction as the local shift of neighboring tracked cells. If the proposed track does not align with the local shift of neighboring tracked cells, then the track is terminated.

SNR calculation

268 Since there is no standard for defining signal-to-noise ratio (SNR) in fluorescence imaging (Zhu

$$
S N R=10 * \log \left(\frac{P_{\text {signal }}}{P_{\text {noise }}}\right)
$$

271 Where we defined $P_{\text {signal }}$ as the average signal (meaningful input) and $P_{\text {noise }}$ as the standard

272 deviation of the background noise. However, since we have no reference image to define what

273 perfect signal is in any raw dataset, we defined our signal to be any pixels above a certain value $274 j$ and noise to be any pixels below that value.

$$
\widehat{S N R}_{j}=10 * \log \left(\frac{P_{\text {signal } \geq j}}{P_{\text {noise }<j}}\right)
$$

276 Where $P_{\text {signal }}$ is defined as the mean of all values above $j$, and $P_{\text {noise }}$ is defined as the standard 277 deviation of all values below $j$. Since $j$ would otherwise be arbitrarily determined, we chose to 
calculate $j$ from the entire image volume using Otsu threshold for binarization (Otsu, 1979), providing us with a reference free metric of SNR.

\section{Statistical analysis}

All statistical analysis was performed using Python statsmodels and scipy libraries. $\mathrm{N}$ represents the number of animals used in each experiment, unless otherwise noted. Data are reported as mean \pm SEM or median \pm SEM as indicated, and $p<0.05$ was considered statistically significant. Level of significance is marked on figures as follows: * denotes $p<0.05$; ${ }^{* *}$ denotes $\mathrm{p}<0.01 ;{ }^{* * *}$ denotes $\mathrm{p}<0.001$.

\section{Code availability}

Packaged software code for Oligo-Track is readily available at github.com/Bergleslab/Xu Bergles 2021 Oligo Track along with instructions for use. The algorithm is prepared to work independent of Linux and Windows operating systems, with minimum Python 3.6.

\section{RESULTS}

\section{Quantifying oligodendrocyte dynamics in vivo using CNN-assisted cell tracking}

To visualize individual oligodendrocytes in the cerebral cortex, cranial windows were surgically implanted in mice that express EGFP under control of the Mobp promoter/enhancer (Hughes et al., 2018; Orthmann-Murphy et al., 2020) (Figure 1A). Using two-photon microscopy, the somas and cytosolic processes of oligodendrocytes could be resolved up to a depth of $\sim 400 \mu \mathrm{m}$ from the pial surface (Figures 1B,C), providing the means to quantify changes in both the number and distribution of oligodendrocytes over weeks to months with repeated imaging. The dramatic increase in density of oligodendrocytes with depth (Figure 1C) presents challenges for unambiguous identification and increases the time necessary to mark and track cell positions 
throughout a time series. To overcome this quantitative challenge, we trained two sequential CNNs employing a UNet architecture (Supplementary Figure S1), which we termed Seg-CNN and Track-CNN, to follow oligodendrocytes in vivo during repetitive bouts of imaging over many weeks (Figure 2A).

Images were first acquired over a $850 \mu \mathrm{m} \times 850 \mu \mathrm{m} \times 550 \mu \mathrm{m}$ volume and then registered across time using ImageJ's correct 3D drift plugin (Schindelin et al., 2012; Parslow et al., 2014) to adjust for small offsets. Seg-CNN was then used to perform semantic segmentation to identify the position of all oligodendrocyte cell bodies within the imaging volume at each timepoint in the timeseries. This process was completed sequentially on $256 \times 256 \times 64$ voxel volumes that were adaptively cropped with $50 \%$ spatial overlap to reduce the amount of computer memory required to perform the computations (Figure 2B). The resulting binary segmentations were then re-stitched to create a stacked timeseries. Image stacks from sequential time points were then analyzed using Track-CNN, which employs a "seed-based" inference approach to determine whether any specific cell of interest exists in a subsequent timepoint. For all comparisons, we defined a tracked cell (or cell track), as a set of locations where a binary object was determined to be the same cell over subsequent timepoints by an algorithm or human researcher. The displacement vector for any cell thus starts at a soma on timepoint $t$ and ends at the same tracked soma on $t+n$. Cell identification in Track-CNN is accomplished by providing a three-channel input to the CNN, which includes (1) a crop of raw fluorescence from timepoint $t$ centered around a cell of interest, (2) a binary seed-mask that emphasizes the current cell of interest, and (3) a crop of raw fluorescence from timepoint $t+1$ that is centered around the cell on $t$. This allows the CNN output to be a volumetric segmentation of the same cell on timepoint $t+1$, given a masked cell of interest on timepoint $t$ (Figure 3A). Additional post-processing was performed using local tissue movement vectors to detect gross errors in tracking between sequential timepoints (Figures 3B, C). This postprocessing used the observation that the displacement vector for any cell can be predicted 
within 10-pixel accuracy using the average displacement vectors of the nearest five tracked cells (Figure 3C). Thus, any cells with displacement vectors that varied drastically from predicted vectors, calculated from nearest neighbor tracks, could be classified as incorrect associations. Overall, during training, Seg-CNN performance plateaued after $\sim 30$ epochs, demonstrating accurate segmentation of cell somas relative to ground truth (Jaccard overlap index $~ 0.7)$ and detection of cells across all volumes (95\% sensitivity, 91\% precision; Supplementary Figure S2A). Track-CNN performance plateaued after $\sim 5$ epochs with highly accurate track associations (98\% accuracy, 99\% sensitivity, and 99\% precision; Supplementary Figure S2B).

To determine if this CNN-based method outperforms a heuristic cell tracking method that employs similarity metrics and local tissue movement modeling, similar to the post-processing mentioned above, we tested both algorithms for their ability to extract biological trends of spontaneous cell regeneration in the cuprizone model of demyelination (Chang et al., 2012; Baxi et al., 2017; Hughes et al., 2018; Orthmann-Murphy et al., 2020). In this model, mice are fed cuprizone for three weeks, resulting in loss of $>95 \%$ of oligodendrocytes in the upper layers of cortex, which are progressively regenerated as the mice are returned to a normal diet (Figure 4A). Both CNN and heuristic models detected the general trend of cell loss during the first three weeks of cuprizone treatment and subsequent oligodendrogenesis during recovery, as assessed relative to human counting (Figure 4B). However, closer examination revealed that Oligo-Track provided a more accurate accounting of cell dynamics. In particular, the heuristic 350 method greatly mis-identified existing cells as being newly formed (Figure 4C), suggesting 351 disrupted tracking. This conclusion was further supported by the increased number of wrongly

352 terminated cell tracks by the heuristic algorithm at each timepoint (Figure 4D), suggesting that

353 the heuristic approach often failed to identify existing oligodendrocytes in subsequent time

354 points. We also assessed the difference in track length (persistence of cells during the time 355 series) between ground truth and machine outputs (Figure 4E). Positive values in this plot 
indicate under-tracking, where the machine failed to track a cell in subsequent timepoints, while negative values indicate over-tracking, where the machine tracked a cell onto additional timepoints despite cell elimination determined in the ground truth. This graph reveals that TrackCNN markedly reduced the total rate of over-tracked segments errors two-fold from the heuristic algorithm (Figure 4F). Moreover, the severe error rate (under or over-tracking for $>1$ timepoint) decreased almost five-fold. Together, these findings indicate that Oligo-Track provides substantial benefits for following oligodendrocytes in longitudinal 3D imaging datasets.

\section{CNN-based analysis retains tracking ability despite changes in image quality}

Many factors can influence image quality in vivo, limiting the ability to accurately assess cell

372 invariant, but struggled with up-sampling beyond two-fold (Figure 5A,B) showing that, optimally, 373 input data should be scaled to the same $0.83 \mu \mathrm{m} /$ pixel $X Y$ and $3 \mu \mathrm{m} /$ pixel in $Z$ resolution as the 374 training dataset. volumes, one from a mouse with an optimal cranial window, and one from a mouse with a

378 window that had not yet become optically clear. The obscured window reduced the detection of

379 fluorescence at lower cortical depths. Our average SNR metric clearly delineated the depth380 dependent decay of image quality, as the SNR in maximum projections of the obscured volume dropped rapidly after a depth of $200 \mu \mathrm{m}$ (Figure 5C,D). This image quality decay was verified 
382 visually, and while Seg-CNN still generalized and was able to identify oligodendrocyte somata in

383 deeper layers despite the reduction in SNR, it was clear that many cells were obscured from

384 view from both machine and human trackers (Figure 5C). By visual assessment, we set a

385 threshold of SNR $\sim 1.5 \mathrm{~dB}$ as a limit under which image quality becomes a concern for Oligo-

386 Track analysis. Fluctuations in SNR between timepoints can lead to disrupted tracking as cells

387 are arbitrarily obscured and falsely labelled as terminated or newly formed. This threshold was

388 incorporated into our pipeline and offers users a warning during implementation of the algorithm.

389 Seg-CNN was also able to avoid some fluorescent, non-cellular components or weak

390 cellular autofluorescence associated with cells other than oligodendrocytes, which can be

391 difficult for non-deep learning approaches (Supplementary Figure 3A). However, the

392 overwhelming density of brightly autofluorescent debris, such as lipofuscin found near the pial

393 surface, were sometimes detected as false positives by Seg-CNN (Supplementary Figure 3B).

394 We suggest that researchers using this software avoid areas with dense debris or lipofuscin, or

395 at least exclude these regions from analysis, although this can be difficult when imaging in aged

396 tissue (Moreno-García et al., 2018; A. Yakovleva et al., 2020). We also determined that while

397 low imaging power impairs cell detection, post-hoc adjustments of the intensity histogram

398 towards higher values recovered some undetected cells (Supplementary Figure 3C). Finally, we

399 found that Track-CNN was robust to some variations in noise and motion blur. This was

400 assessed by applying sequentially larger standard deviations of noise $(10,40,50)$ and

401 increasing the rotation range of random motion artifacts $(4,6,10$ degrees) using the Torchio

402 python library (Pérez-García et al., 2021) (Supplementary Figure 3D,E). Together, this analysis

403 shows that Oligo-Track can maintain performance despite changes in environmental variables

404 that affect the distribution of the data. Moreover, we demonstrated that pre-processing of input

405 data, such as intensity adjustments and the exclusion of regions with high debris or low SNR,

406 can reduce instances of inaccurate tracking. 


\section{CNN detects layer-specific suppression of oligodendrogenesis at extended depth}

409

410

411

412

413

414

415

416

417

418

419

420

421

422

423

424

425

426

427

428

429

430

431

432

433

To assess the capacity of our pipeline to extract biological trends, we used the fully automated system to analyze oligodendrocyte dynamics for up to 12 weeks in cuprizone treated and nontreated control mice. As anticipated, cuprizone treatment resulted in a predictable time course of oligodendrocyte degeneration and subsequent regeneration after mice were no longer exposed to the drug, while control mice gradually added oligodendrocytes over several weeks (Orthmann-Murphy et al., 2020) (Figures 6A,B, Videos 1 and 2). Moreover, when cells were segregated into $100 \mu \mathrm{m}$ thick blocks from the pial surface, greater suppression of oligodendrocyte regeneration was observed in the deeper layers of the cortex (Figure 6C), as reported previously (Orthmann-Murphy et al., 2020). The sensitive detection of Oligo-Track allowed rapid extension of the analysis by another $100 \mu \mathrm{m}(300-400 \mu \mathrm{m}$ block), revealing that regeneration was even less efficient than in the area above, providing further evidence of the depth dependent decline in oligodendrocyte regeneration in the somatosensory cortex.

It is possible that the higher demand for oligodendrocyte regeneration in deeper cortical layers outstrips the regenerative capacity of OPCs (Hughes et al., 2013; Streichan et al., 2014). If the extent of oligodendrogenesis is limited by the availability of local cues or accumulation of myelin debris, then newly generated cells should preferentially appear in regions with lower initial oligodendrocyte density (and lower oligodendrocyte death) (Orthmann-Murphy et al., 2020). Our prior studies indicate that new oligodendrocytes do not regenerate in locations where previous cells had died, suggesting possible inhibition of proliferation by myelin debris after cell death (Lampron et al., 2015; Gruchot et al., 2019). As a measure of sparsity, we calculated the average distance from each cell to its five nearest neighbors. We limited our analysis to the first $300 \mu \mathrm{m}$ of the cortex to avoid errors in sparsity calculations due to the lack of tracked nearest-neighbor cells past $400 \mu \mathrm{m}$ depth. Given this measure, we found that there was no strong correlation between sparsity, cell death or regeneration (Figures 6D,E and Video 3), suggesting that cell death and regeneration are not strongly influenced by local oligodendrocyte 
density at baseline. Rather, global gradients of inhibitory factors such as cytokines released by astrocytes, which become persistently reactive in deeper layers of the cortex after cuprizone mediated demyelination (Orthmann-Murphy et al., 2020), may inhibit oligodendrocyte precursor cell differentiation (Skripuletz et al., 2008; Zhang et al., 2010; Su et al., 2011; Chang et al., 2012; Kirby et al., 2019).

\section{Volumetric segmentation enables identification of newly born oligodendrocytes}

Oligodendrocytes undergo dramatic morphological changes as they transition from progenitors to mature myelinating cells, accompanied by an elaboration of myelin forming processes and changes in soma size (Kuhn et al., 2019). To quantify the time course of these somatic changes, we analyzed volumetric morphological data provided by Oligo-Track, from longitudinal imaging datasets where the birth date of newly formed oligodendrocytes was known. We limited our investigation to the first $300 \mu \mathrm{m}$ of the cortex as tissue refraction often reduced brightness of cells in deeper cortical layers, resulting in inaccurate measurement of cell soma volume from dim fluorescence. This analysis revealed oligodendrocyte soma size was highly correlated with cell age. Most newly formed oligodendrocytes had larger cell bodies than stable cells at any timepoint across all depths (Figure 7A and Video 4). Projecting this across cell age, the soma volume of newly formed oligodendrocytes decayed exponentially over subsequent weeks from first appearance (Figure 7B,C; p $<0.001 @ 1$ week, p=0.027 @ 2 weeks; Kruskal-Wallis test with Dunn's post-hoc analysis). Moreover, the average volume of newly generated cells, postcuprizone injury, was significantly higher compared to stable mature cells in control animals up to 3 weeks after oligodendrogenesis (Figure 7D; $1.6 \pm 0.04$ fold change $p<0.001$ and $D=1.29 @$ 1 week, $1.4 \pm 0.04$ fold change $p<0.001$ and $D=0.85 @ 2$ weeks, $1.2 \pm 0.03$ fold change $p<0.001$ and $D=0.33 @ 3$ weeks, $1.0 \pm 0.03$ fold change $p=0.39$ and $D=0.07 @ 4$ weeks; Kruskal-Wallis test with Dunn's post-hoc analysis and Cohen's effect size calculation). To confirm that this size difference is not associated with cuprizone induced changes, we also 
compared the volume of spontaneously generated oligodendrocytes in control animals with their stable counterparts and found that newly formed cells also had significantly larger cell somata

(Figure 7D; $1.7 \pm 0.09$ fold change $p<0.001$ and $D=1.22 @ 1$ week, $1.4 \pm 0.07$ fold change $p<0.001$ and $D=1.1 @ 2$ weeks, $1.3 \pm 0.06$ fold change $p<0.001$ and $D=0.54$ @ 3 weeks, $1.0 \pm$ 0.05 fold change $p=0.28$ and $D=0.09 @ 4$ weeks; Kruskal-Wallis test with Dunn's post-hoc analysis and Cohen's effect size calculation). Thus, the increased soma size of newly formed oligodendrocytes is an innate biological phenomenon, rather than a response to cuprizone exposure. assessed the predictive power of cell soma size as an indicator of cell age. To examine the probability that a cell soma of a certain volume is exactly a certain age or within a range of ages, we plotted the kernel density estimate (KDE) for each distribution of soma volumes at different

472 timepoints (Figure 7E). The KDE offers a normalized estimate of the probability density function

473 such that we can visualize the probability of multiple conditions simultaneously. For example,

474 we observed that a cell with a soma volume greater than $5000 \mu^{3}$ has an almost $100 \%$ chance 475 of being exactly 1 week old from time of differentiation. Similarly, cell somata between the range 476 of $3500-5000 \mu m^{3}$ are most likely less than 2 weeks old, while somata larger than $3000 \mu m^{3}$

477 are likely newly generated cells within the first 3 weeks post-differentiation (Figure 7E). Finally, 478 by comparing the mean soma volume of stable control oligodendrocytes to newly formed cells 479 at multiple timepoints, we also confirmed the statistical significance of the predictive relationship 480 between soma volume and cell age (Figure 7F; $p<0.001$ all comparisons; 1 -way ANOVA with 481 Tukey's Honest Significant Difference post-hoc test).

483 Oligodendrocyte death can be predicted from soma size

484 Oligodendrocyte death is typically preceded by nuclear condensation and shrinkage of the soma 485 (Bortner and Cidlowski, 2002; Miller and Zachary, 2017). To determine if the soma size analysis 
could also be used to predict whether an oligodendrocyte will later degenerate, we plotted the

487 soma volumes of all cells later observed to degenerate. After multiple weeks of cuprizone

488 treatment, the median soma volume of all cells shrank significantly (Figures 8A-C, Video 4;

$489 \mathrm{p}<0.001 @ 1$ week, 2 weeks and 3 weeks), consistent with the high degree of oligodendrocyte

490 degeneration observed in the cortex. When compared to oligodendrocytes at comparable

491 timepoints in control mice, soma size was also significantly smaller after extended cuprizone

492 treatment (Figure 8D; $0.83 \pm 0.013$ fold change $p<0.001$ and $D=0.26 @ 1$ week, $0.73 \pm 0.013$

493 fold change $p<0.001$ and $D=0.65 @ 2$ weeks, $0.6 \pm 0.015$ fold change $p<0.001$ and $D=1.1 @ 3$

494 weeks; Kruskal-Wallis test with Dunn's post-hoc analysis and Cohen's effect size calculation),

495 consistent with progression to apoptosis. Given the large statistical power when sampling

496 thousands of cells, we additionally defined a significant difference in soma volume as one

497 having a medium to large effect size ( $>0.5$ Cohen's D), which only occurred at 2 and 3 weeks of

498 cuprizone treatment. Assessing the predictive power of soma volume again, we attempted to

499 predict the likelihood that a cell would die within the next subsequent week given that the cell is

500 smaller than a certain soma volume. While not as striking as the predictive power for newly

501 formed oligodendrocytes, the probability that cells with somata below $500 \mu \mathrm{m}^{3}$ would disappear

502 within one week was over $90 \%$ (Figures 8E,F). Together, this analysis reveals that the size of

503 oligodendrocyte somata calculated using deep neural networks can be used to predict, without

504 prior or later longitudinal imaging data, whether a cell was recently generated and whether it is

505 likely to degenerate.

506

507

508 DISCUSSION

509 To facilitate analysis of oligodendrocyte dynamics in the adult brain we designed Oligo-Track, a

510 deep learning pipeline that uses two sequential CNNs to allow cell tracking in volumetric

511 imaging datasets. This methodology provides a substantial improvement over traditional 
512 imaging informatics approaches as it was faster, less subject to user bias and less influenced by

513 factors that commonly deteriorate image quality, allowing reliable automated cell tracking over

514 time series spanning multiple weeks. This automated volumetric analysis enabled us to increase

515 the number of oligodendrocytes analyzed in deeper layers of the mouse cortex and to identify

516 newly formed oligodendrocytes and those that are in the process of degenerating simply based

517 on soma size at a single time point without longitudinal tracking information.

518 This CNN tracking pipeline follows a two-step approach to optimize multi-object tracking

519 (MOT). We first setup a detection step, where oligodendrocytes are identified in a volume,

520 followed by an association step, to link tracked cells across time frames (Ciaparrone et al.,

521 2020). Unlike other deep learning MOT approaches, which often only use CNNs to generate

522 bounding boxes or extract features (Ciaparrone et al., 2020), we employed two sequential

523 CNNs that both performed semantic segmentation in the MOT detection and association stages

524 (Seg-CNN and Track-CNN, respectively). The output of this pipeline provides not only the

525 location of all tracked cells, but also the volume of each cell soma. This volumetric tracking was

526 made possible by training our association network (Track-CNN) with a seed-based learning

527 approach. Previous studies have shown that, when given input data containing several cells,

528 one can mark cells of interest with a binary mask, or "seed", to draw the attention of CNNs (Xu

529 et al., 2019). This forces a semantic classifier to not only learn to identify oligodendrocyte

530 somas, but also to identify the somas of individually marked cells of interest across different

531 timepoints.

532 From a computational standpoint, there are several advantages to this automated

533 approach. Roughly estimating the time for manual analysis with syGlass, a 3D virtual reality

534 based visualization tool, we found that a 10-week, 10-timepoint dataset with a size of $800 \times 800$

$535 \times 300 \mu \mathrm{m}$ per timepoint would take a researcher approximately six hours to identify and track all

536 oligodendrocytes within this volume. This estimate only considers the time to place point

537 coordinates and does not include the considerable additional time it would take to trace every 
voxel to generate volumetric segmentations. This estimate also does not consider how much longer manual analysis would take without access to specialized VR software (e.g. syGlass). By comparison, Oligo-Track requires $\sim 20$ minutes for Seg-CNN segmentation ( 2 minutes per

541 timepoint) and $\sim 25-35$ minutes for Track-CNN associations for the same volume across 10

542 timepoints, for a total analysis time of $45-55$ minutes, more than six times faster than achieved 543 with VR-assisted manual tracking, just for cell identification. This processing time is also purely

544 computational, so manual labor time is reduced to almost zero, and offers fully volumetric

545 segmentations. Total runtime will vary depending on cell density, number of timepoints, the size of volumes during inference and the exact computer configuration. analysis approach. Losing dimensionality can be extremely detrimental to quantification speed and accuracy, as cells can often lie on top of one another or shift in unpredictable ways that can be missed if viewing 4D data in lower dimensional space. As many researchers do not yet have access to 4D visualization/tracking tools, Oligo-Track standardizes the approach to longitudinal cell tracking, removing the reliance on specialized proprietary software and reducing tracking inconsistencies between individuals. decision to use deep learning as an underlying analytic framework comes with additional considerations. Deep learning is often criticized for its "black box" nature, as researchers are

557 unable to understand the intricate decision-making process of millions of weighted connections

558 in a CNN, resulting in sometimes unpredictable behavior (Heaven, 2019; Yampolskiy, 2019).

559 For example, as we see in our own network, it was difficult to define the exact level of debris 560 avoidance that the neural network was capable of, and why certain debris were more likely to be 561 identified as false positives. This variability could be addressed in future work by data 562 augmentation, whereby data containing high levels of real or synthetic debris could be 563 introduced during CNN training. Currently, we partially addressed the unpredictability of deep 
learning by using VR-based 4D manual curation post-CNN analysis to ensure accuracy in unpredictable scenarios. We also used these post-hoc manually curated datasets to further improve the CNNs, highlighting a major advantage of deep learning approaches. CNN models are extraordinarily data hungry and can be continuously improved with new training data that help generalize to new imaging conditions (Klabjan and Zhu, 2020). For instance, while Oligo-

569 Track has only been trained on cells up to $400 \mu \mathrm{m}$ depth in the cortex, it will be possible to

570 further train these networks to imaging conditions in deeper cortical layers. This training

571 advantage is not available for traditional algorithms that may require extensive manual fine-

572 tuning for extrapolation to slight variations in imaging conditions. features of cells that express fluorescent proteins, our pretrained CNN can serve as a basis for additional tool development, in which transfer learning is used to adapt this model to other cell types, where ground truth training data may not be readily available.

Automated quantitative tools will play a growing, critical role in the age of big data that is spurned by advances in biological imaging technologies. Of note for oligodendrocyte biology,

584 three photon imaging promises to take us deeper in vivo (Horton et al., 2013; Lecoq et al., 585 2019), allowing us to examine the dynamics of these myelinating cells in layers 5 and 6 of the 586 cortex and perhaps even into the white matter of the corpus callosum. Additionally, block-face 587 imaging presents us with the opportunity to examine distributions of oligodendrocytes across the 588 entire mouse brain, correlating myelination patterns with neuron type and brain region (Ragan et al., 2012; Amato et al., 2016; Winnubst et al., 2019). To match the scale of these imaging 
technologies, an important extension of the current work is to extract not only positional

591 information about cells in vivo, but also the entire structure of cells. For oligodendrocytes, that

592 means the soma, cytosolic branches, and individual myelin sheaths formed by each cell. As

593 highlighted in this study, gaining quantitative access to even a single parameter, such as soma

594 volume, can greatly extend biological understanding, allowing robust predictions to be made

595 with limited data. Here, the strong correlations we observed between soma size, age, and

596 survival provide us with a tool to infer the regenerative capacity of oligodendrocytes on fixed

597 timepoint experiments acquired from individual tissue sections or from block-face imaging

598 (Ragan et al., 2012). By extension, having access to the complete morphological structures of

599 thousands of oligodendrocytes in the brain would enable us to assess complex region-specific

600 differences in adaptive myelination, regenerative capacity and survival across the brain in mice 601 subjected to different interventions. challenge, which not only facilitates analysis of oligodendrocyte dynamics, but also extends our quantitative limits to extract novel insight into regional differences in regenerative capacity and allows predictions to be made about future behaviors. Having access to more cellular features and dynamics will bring us closer to understanding the events that underlie myelin regeneration

611 that will aid in the discovery of therapeutics for treating demyelinating diseases. 


\section{ACKNOWLEDGMENTS}

617 We thank Dr. M. Pucak and N. Ye for technical assistance, T. Shelly for machining expertise,

618 and members of the Bergles laboratory for discussions. Y.K.T.X. was supported by a fellowship

619 from the Johns Hopkins University Kavli Neuroscience Discovery Institute and C.C. was

620 supported by a National Science Foundation Graduate Research Fellowship. Funding was

621 provided by NIH BRAIN Initiative grant R01 RF1MH121539, a Collaborative Research Center

622 Grant from the National Multiple Sclerosis Society and the Dr. Miriam and Sheldon G Adelson

623 Medical Research Foundation.

\section{Author contributions}

626 Y.K.T.X. model design, conceptualization, data curation, formal analysis, supervision, validation,

627 investigation, visualization, methodology, writing - original draft, writing - review and editing.

628 C.C. conceptualization, data curation, supervision, methodology, writing - original draft, writing

629 - review and editing. J.S. methodology, supervision, investigation, writing - original draft, writing

630 - review and editing. D.E.B. conceptualization, Resources, Supervision, Funding acquisition,

631 Investigation, Methodology, Writing - original draft, Project administration, Writing - review and

632 editing.

634 Conflict of Interest

635 The authors declare that the research was conducted in the absence of any commercial or

636 financial relationships that could be construed as a potential conflict of interest.

\section{REFERENCES}

Acton, S. T., Wethmar, K., and Ley, K. (2002). Automatic Tracking of Rolling Leukocytes in Vivo. Microvascular Research 63, 139-148. doi:10.1006/mvre.2001.2373. 
Amato, S. P., Pan, F., Schwartz, J., and Ragan, T. M. (2016). Whole Brain Imaging with Serial Two-Photon Tomography. Front Neuroanat 10. doi:10.3389/fnana.2016.00031.

A. Yakovleva, M., Sh. Radchenko, A., B. Feldman, T., A. Kostyukov, A., M. Arbukhanova, P., A. Borzenok, S., et al. (2020). Fluorescence characteristics of lipofuscin fluorophores from human retinal pigment epithelium. Photochemical \& Photobiological Sciences 19, 920-930. doi:10.1039/C9PP00406H.

Baxi, E. G., DeBruin, J., Jin, J., Strasburger, H. J., Smith, M. D., Orthmann-Murphy, J. L., et al. (2017). Lineage tracing reveals dynamic changes in oligodendrocyte precursor cells following cuprizone-induced demyelination. Glia 65, 2087-2098. doi:10.1002/glia.23229.

Berg, S., Kutra, D., Kroeger, T., Straehle, C. N., Kausler, B. X., Haubold, C., et al. (2019). ilastik: interactive machine learning for (bio)image analysis. Nature Methods 16, 1226-1232. doi:10.1038/s41592-019-0582-9.

Bergles, D. E., and Richardson, W. D. (2015). Oligodendrocyte Development and Plasticity. Cold Spring Harb Perspect Biol 8, a020453. doi:10.1101/cshperspect.a020453.

Bhardwaj, R. D., Curtis, M. A., Spalding, K. L., Buchholz, B. A., Fink, D., Björk-Eriksson, T., et al. (2006). Neocortical neurogenesis in humans is restricted to development. PNAS 103, 12564-12568. doi:10.1073/pnas.0605177103.

Bortner, C. D., and Cidlowski, J. A. (2002). Apoptotic volume decrease and the incredible shrinking cell. Cell Death \& Differentiation 9, 1307-1310. doi:10.1038/sj.cdd.4401126.

Chang, A., Staugaitis, S. M., Dutta, R., Batt, C. E., Easley, K. E., Chomyk, A. M., et al. (2012). Cortical remyelination: A new target for repair therapies in multiple sclerosis. Annals of Neurology 72, 918-926. doi:10.1002/ana.23693.

Ciaparrone, G., Sánchez, F. L., Tabik, S., Troiano, L., Tagliaferri, R., and Herrera, F. (2020). Deep Learning in Video Multi-Object Tracking: A Survey. Neurocomputing 381, 61-88. doi:10.1016/j.neucom.2019.11.023.

El Waly, B., Macchi, M., Cayre, M., and Durbec, P. (2014). Oligodendrogenesis in the normal and pathological central nervous system. Front. Neurosci. 8. doi:10.3389/fnins.2014.00145.

Giovannucci, A., Friedrich, J., Gunn, P., Kalfon, J., Brown, B. L., Koay, S. A., et al. (2019). CalmAn an open source tool for scalable calcium imaging data analysis. eLife 8, e38173. doi:10.7554/eLife.38173.

Gruchot, J., Weyers, V., Göttle, P., Förster, M., Hartung, H.-P., Küry, P., et al. (2019). The Molecular Basis for Remyelination Failure in Multiple Sclerosis. Cells 8. doi:10.3390/cells8080825.

Heaven, D. (2019). Why deep-learning Als are so easy to fool. Nature 574, 163-166. doi:10.1038/d41586-019-03013-5. 
Holtmaat, A., de Paola, V., Wilbrecht, L., Trachtenberg, J. T., Svoboda, K., and Portera-Cailliau, C. (2012). Imaging neocortical neurons through a chronic cranial window. Cold Spring Harb Protoc 2012, 694-701. doi:10.1101/pdb.prot069617.

Horton, N. G., Wang, K., Kobat, D., Clark, C. G., Wise, F. W., Schaffer, C. B., et al. (2013). In vivo three-photon microscopy of subcortical structures within an intact mouse brain. Nature Photonics 7, 205-209. doi:10.1038/nphoton.2012.336.

Hughes, E. G., Kang, S. H., Fukaya, M., and Bergles, D. E. (2013). Oligodendrocyte progenitors balance growth with self-repulsion to achieve homeostasis in the adult brain. Nat Neurosci 16, 668-676. doi:10.1038/nn.3390.

Hughes, E. G., Orthmann-Murphy, J. L., Langseth, A. J., and Bergles, D. E. (2018). Myelin remodeling through experience-dependent oligodendrogenesis in the adult somatosensory cortex. Nature Neuroscience 21, 696-706. doi:10.1038/s41593-0180121-5.

Jaccard, P. (1912). The Distribution of the Flora in the Alpine Zone.1. New Phytologist 11, 3750. doi:10.1111/j.1469-8137.1912.tb05611.x.

Kirby, L., Jin, J., Cardona, J. G., Smith, M. D., Martin, K. A., Wang, J., et al. (2019). Oligodendrocyte precursor cells present antigen and are cytotoxic targets in inflammatory demyelination. Nature Communications 10, 3887. doi:10.1038/s41467019-11638-3.

Klabjan, D., and Zhu, X. (2020). Neural Network Retraining for Model Serving. arXiv:2004.14203 [cs, stat]. Available at: http://arxiv.org/abs/2004.14203 [Accessed October 31, 2020].

Kuhn, S., Gritti, L., Crooks, D., and Dombrowski, Y. (2019). Oligodendrocytes in Development, Myelin Generation and Beyond. Cells 8. doi:10.3390/cells8111424.

Lampron, A., Larochelle, A., Laflamme, N., Préfontaine, P., Plante, M.-M., Sánchez, M. G., et al. (2015). Inefficient clearance of myelin debris by microglia impairs remyelinating processes. J Exp Med 212, 481-495. doi:10.1084/jem.20141656.

Larson, V. A., Mironova, Y., Vanderpool, K. G., Waisman, A., Rash, J. E., Agarwal, A., et al. (2018). Oligodendrocytes control potassium accumulation in white matter and seizure susceptibility. Elife 7. doi:10.7554/eLife.34829.

Lecoq, J., Orlova, N., and Grewe, B. F. (2019). Wide. Fast. Deep: Recent Advances in Multiphoton Microscopy of In Vivo Neuronal Activity. J. Neurosci. 39, 9042-9052. doi:10.1523/JNEUROSCI.1527-18.2019.

Loshchilov, I., and Hutter, F. (2019). Decoupled Weight Decay Regularization. arXiv:1711.05101 [cs, math]. Available at: http://arxiv.org/abs/1711.05101 [Accessed October 30, 2020].

Lugagne, J.-B., Lin, H., and Dunlop, M. J. (2020). DeLTA: Automated cell segmentation, tracking, and lineage reconstruction using deep learning. PLoS Comput Biol 16. doi:10.1371/journal.pcbi.1007673. 
Marshall, L., Call, C., Bergles, D. E., and Morehead, M. Quicker, cost effective tracking of 4D data.

Miller, M. A., and Zachary, J. F. (2017). Mechanisms and Morphology of Cellular Injury, Adaptation, and Death. Pathologic Basis of Veterinary Disease, 2-43.e19. doi:10.1016/B978-0-323-35775-3.00001-1.

Ming, G., and Song, H. (2011). Adult Neurogenesis in the Mammalian Brain: Significant Answers and Significant Questions. Neuron 70, 687-702. doi:10.1016/j.neuron.2011.05.001.

Moreno-García, A., Kun, A., Calero, O., Medina, M., and Calero, M. (2018). An Overview of the Role of Lipofuscin in Age-Related Neurodegeneration. Front. Neurosci. 12. doi:10.3389/fnins.2018.00464.

Narayanan, R. T., Udvary, D., and Oberlaender, M. (2017). Cell Type-Specific Structural Organization of the Six Layers in Rat Barrel Cortex. Front. Neuroanat. 11. doi:10.3389/fnana.2017.00091.

Neyshabur, B., Bhojanapalli, S., McAllester, D., and Srebro, N. (2017). Exploring Generalization in Deep Learning. arXiv:1706.08947 [cs]. Available at: http://arxiv.org/abs/1706.08947 [Accessed November 1, 2020].

Nguyen, N. H., Keller, S., Norris, E., Huynh, T. T., Clemens, M. G., and Shin, M. C. (2011). Tracking Colliding Cells In Vivo Microscopy. IEEE Transactions on Biomedical Engineering 58, 2391-2400. doi:10.1109/TBME.2011.2158099.

Nketia, T. A., Sailem, H., Rohde, G., Machiraju, R., and Rittscher, J. (2017). Analysis of live cell images: Methods, tools and opportunities. Methods 115, 65-79. doi:10.1016/j.ymeth.2017.02.007.

Orthmann-Murphy, J., Call, C. L., Molina-Castro, G. C., Hsieh, Y. C., Rasband, M. N., Calabresi, P. A., et al. (2020). Remyelination alters the pattern of myelin in the cerebral cortex. elife 9, e56621. doi:10.7554/eLife.56621.

Otsu, N. (1979). A Threshold Selection Method from Gray-Level Histograms. IEEE Transactions on Systems, Man, and Cybernetics 9, 62-66. doi:10.1109/TSMC.1979.4310076.

Pachitariu, M., Stringer, C., Dipoppa, M., Schröder, S., Rossi, L. F., Dalgleish, H., et al. (2017). Suite2p: beyond 10,000 neurons with standard two-photon microscopy. bioRxiv, 061507. doi:10.1101/061507.

Parslow, A., Cardona, A., and Bryson-Richardson, R. J. (2014). Sample Drift Correction Following 4D Confocal Time-lapse Imaging. J Vis Exp. doi:10.3791/51086.

Paszke, A., Gross, S., Chintala, S., Chanan, G., Yang, E., DeVito, Z., et al. (2017). Automatic differentiation in PyTorch. Available at: https://openreview.net/forum?id=BJJsrmfCZ [Accessed October 30, 2020].

Pérez-García, F., Sparks, R., and Ourselin, S. (2021). TorchIO: a Python library for efficient loading, preprocessing, augmentation and patch-based sampling of medical images in 
deep learning. arXiv:2003.04696 [cs, eess, stat]. Available at: http://arxiv.org/abs/2003.04696 [Accessed January 18, 2021].

Pidhorskyi, S., Morehead, M., Jones, Q., Spirou, G., and Doretto, G. (2018). syGlass: Interactive Exploration of Multidimensional Images Using Virtual Reality Head-mounted Displays. arXiv:1804.08197 [cs]. Available at: http://arxiv.org/abs/1804.08197 [Accessed October 30, 2020].

Ragan, T., Kadiri, L. R., Venkataraju, K. U., Bahlmann, K., Sutin, J., Taranda, J., et al. (2012). Serial two-photon tomography: an automated method for ex-vivo mouse brain imaging. Nat Methods 9, 255-258. doi:10.1038/nmeth.1854.

Ronneberger, O., Fischer, P., and Brox, T. (2015). U-Net: Convolutional Networks for Biomedical Image Segmentation. Available at: https://arxiv.org/abs/1505.04597v1 [Accessed March 7, 2020].

Schindelin, J., Arganda-Carreras, I., Frise, E., Kaynig, V., Longair, M., Pietzsch, T., et al. (2012). Fiji: an open-source platform for biological-image analysis. Nature Methods 9, 676-682. doi:10.1038/nmeth.2019.

Simons, M., and Nave, K.-A. (2016). Oligodendrocytes: Myelination and Axonal Support. Cold Spring Harb Perspect Biol 8. doi:10.1101/cshperspect.a020479.

Skripuletz, T., Lindner, M., Kotsiari, A., Garde, N., Fokuhl, J., Linsmeier, F., et al. (2008). Cortical Demyelination Is Prominent in the Murine Cuprizone Model and Is StrainDependent. The American Journal of Pathology 172, 1053-1061. doi:10.2353/ajpath.2008.070850.

Streichan, S. J., Hoerner, C. R., Schneidt, T., Holzer, D., and Hufnagel, L. (2014). Spatial constraints control cell proliferation in tissues. Proc Natl Acad Sci U S A 111, 55865591. doi:10.1073/pnas.1323016111.

Su, Z., Yuan, Y., Chen, J., Zhu, Y., Qiu, Y., Zhu, F., et al. (2011). Reactive Astrocytes Inhibit the Survival and Differentiation of Oligodendrocyte Precursor Cells by Secreted TNF- $\alpha$. Journal of Neurotrauma 28, 1089-1100. doi:10.1089/neu.2010.1597.

Theer, P., and Denk, W. (2006). On the fundamental imaging-depth limit in two-photon microscopy. J. Opt. Soc. Am. A, JOSAA 23, 3139-3149. doi:10.1364/JOSAA.23.003139.

Van Valen, D. A., Kudo, T., Lane, K. M., Macklin, D. N., Quach, N. T., DeFelice, M. M., et al. (2016). Deep Learning Automates the Quantitative Analysis of Individual Cells in LiveCell Imaging Experiments. PLoS Comput Biol 12. doi:10.1371/journal.pcbi.1005177.

Wang, J., Su, X., Zhao, L., and Zhang, J. (2020). Deep Reinforcement Learning for Data Association in Cell Tracking. Front Bioeng Biotechnol 8. doi:10.3389/fbioe.2020.00298.

Winnubst, J., Bas, E., Ferreira, T. A., Wu, Z., Economo, M. N., Edson, P., et al. (2019). Reconstruction of 1,000 Projection Neurons Reveals New Cell Types and Organization of Long-Range Connectivity in the Mouse Brain. Cell 179, 268-281.e13. doi:10.1016/j.cell.2019.07.042. 
Xu, Y. K. T., Chitsaz, D., Brown, R. A., Cui, Q. L., Dabarno, M. A., Antel, J. P., et al. (2019). Deep learning for high-throughput quantification of oligodendrocyte ensheathment at single-cell resolution. Commun Biol 2, 1-12. doi:10.1038/s42003-019-0356-z.

Yampolskiy, R. V. (2019). Unpredictability of Al. arXiv:1905.13053 [cs]. Available at: http://arxiv.org/abs/1905.13053 [Accessed October 31, 2020].

Zhang, Y., Zhang, J., Navrazhina, K., Argaw, A. T., Zameer, A., Gurfein, B. T., et al. (2010). TGF $\beta 1$ induces Jagged1 expression in astrocytes via ALK5 and Smad3 and regulates the balance between oligodendrocyte progenitor proliferation and differentiation. Glia 58 , 964-974. doi:10.1002/glia.20978.

Zhong, B., Pan, S., Wang, C., Wang, T., Du, J., Chen, D., et al. (2016). Robust IndividualCell/Object Tracking via PCANet Deep Network in Biomedicine and Computer Vision. Biomed Res Int 2016. doi:10.1155/2016/8182416.

Zhou Wang, Bovik, A. C., Sheikh, H. R., and Simoncelli, E. P. (2004). Image quality assessment: from error visibility to structural similarity. IEEE Transactions on Image Processing 13, 600-612. doi:10.1109/TIP.2003.819861.

Zhu, X., Li, X., Li, Z., and Zhu, B. (2012). Study on Signal-to-noise ratio algorithms based on noreference Image quality assessment. in 2012 International Conference on Systems and Informatics (ICSAI2012), 1755-1759. doi:10.1109/ICSAI.2012.6223383.

Zhuang, F., Qi, Z., Duan, K., Xi, D., Zhu, Y., Zhu, H., et al. (2020). A Comprehensive Survey on Transfer Learning. arXiv:1911.02685 [cs, stat]. Available at: http://arxiv.org/abs/1911.02685 [Accessed October 30, 2020]. 


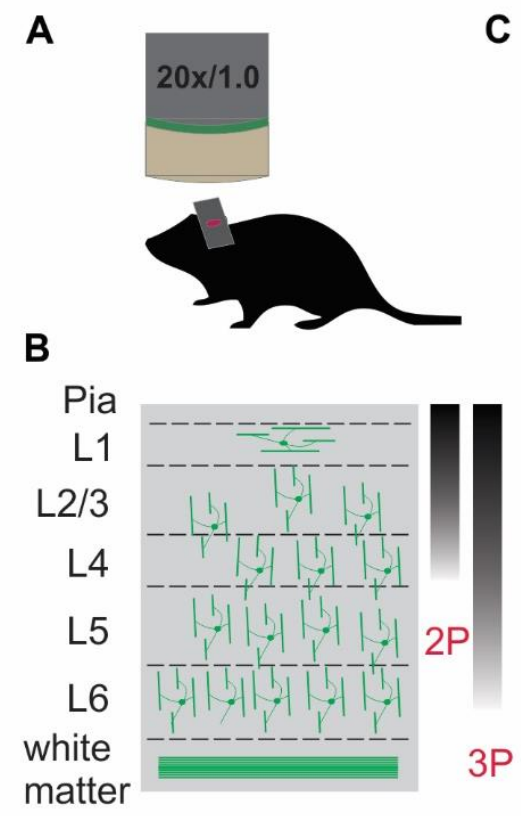

C

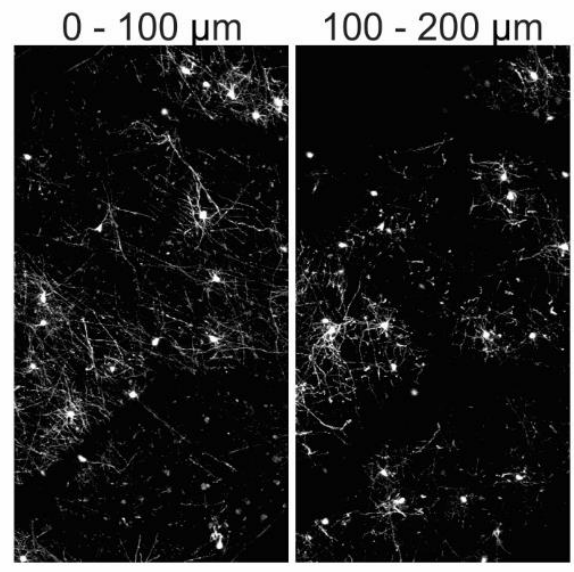

Cortical depth

Figure 1: In vivo imaging of oligodendrocytes. (A) Cranial windows were surgically implanted in adult Mobp-EGFP mice in which only oligodendrocytes express EGFP. (B) Orientation of oligodendrocytes from imaging surface to white matter. Oligodendrocytes in upper cortical layers myelinate horizontally aligned axons, while those in deeper cortical layers are aligned perpendicularly to pial surface. Standard imaging range of two-photon and three-photon microscopy highlighted with approximate gradients (Theer and Denk, 2006; Lecoq et al., 2019) (C) XY maximum projections of $100 \mu \mathrm{m}$ thick volumes at indicated depths $(0-100 \mu \mathrm{m}, 100-$ $200 \mu \mathrm{m}, 200-300 \mu \mathrm{m}, 300-400 \mu \mathrm{m})$. Layer depths as estimated in somatosensory cortex (Narayanan et al., 2017). Oligodendrocyte density increases rapidly with depth, increasing the time needed for manual tracking. Scale bar: $50 \mu \mathrm{m}$. 
A

Oligo-track pipeline

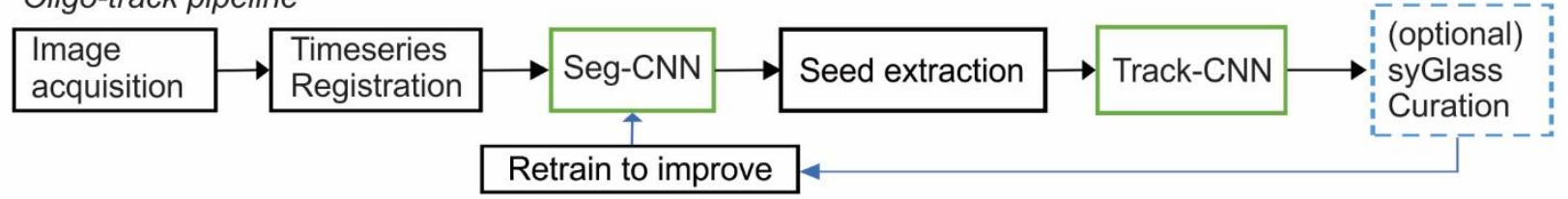

Heurisitic pipeline

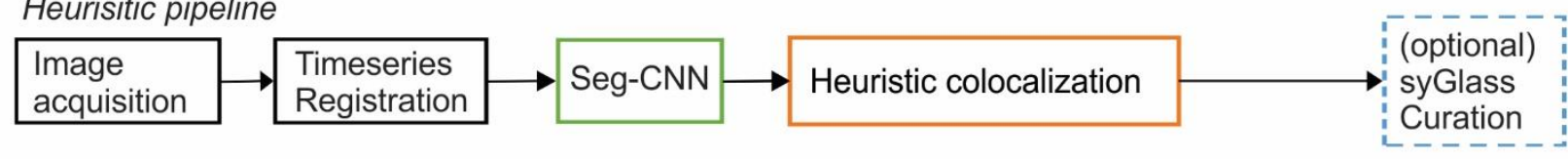

B

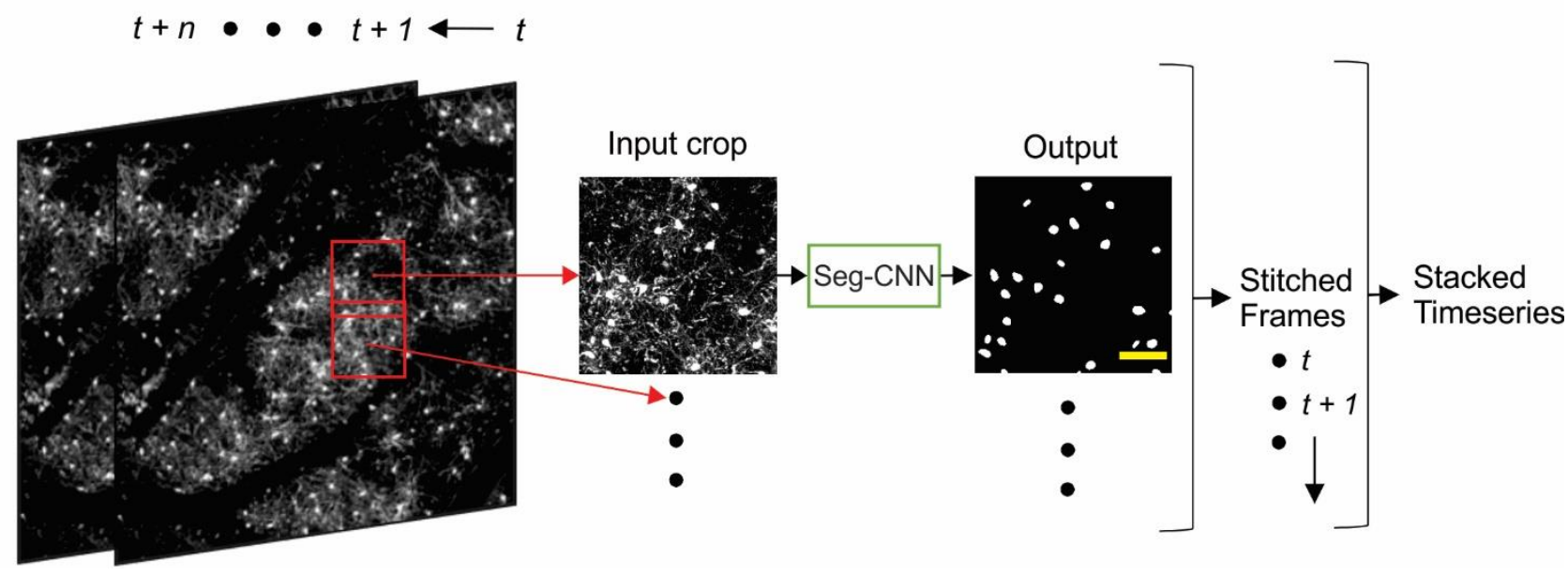

826

827

828

829

830

831

832

833

834

835

836
Figure 2: Computational neural network analysis pipeline. (A) Overview of the sequential CNN multi-object tracking pipeline Oligo-Track (top). CNNs marked in green. Overview of heuristic baseline method (orange) for comparison to Oligo-Track (bottom). Compatibility with optional syGlass curation provides validation of tracking in both pipelines (blue). Curated tracks can also be reintroduced into training pipeline for refinement of CNNs. (B) Seg-CNN preprocessing extracts cropped regions from larger volumes with $50 \%$ overlap for computational efficiency at each timepoint $t$ to $t+n$. Cropped regions are restitched to form timeseries. Scale bar: $50 \mu \mathrm{m}$. 
A

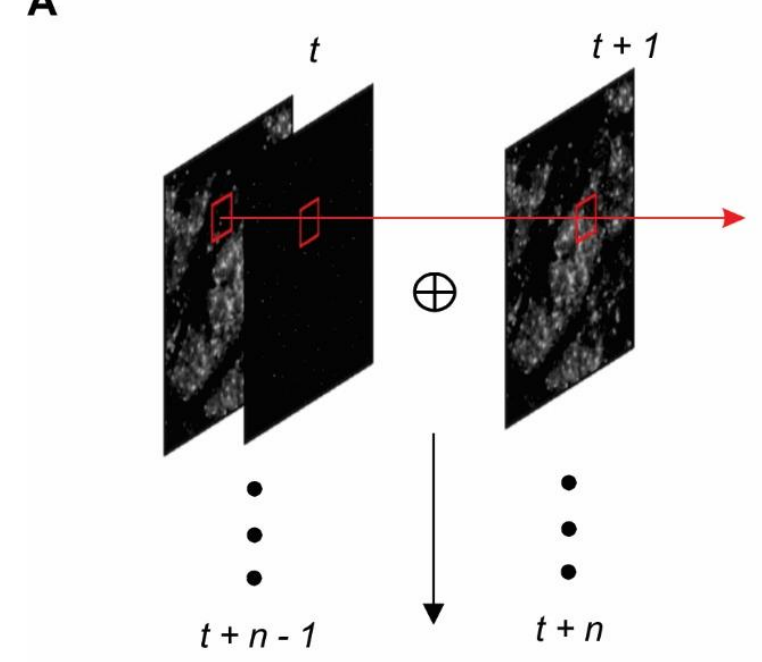

Input

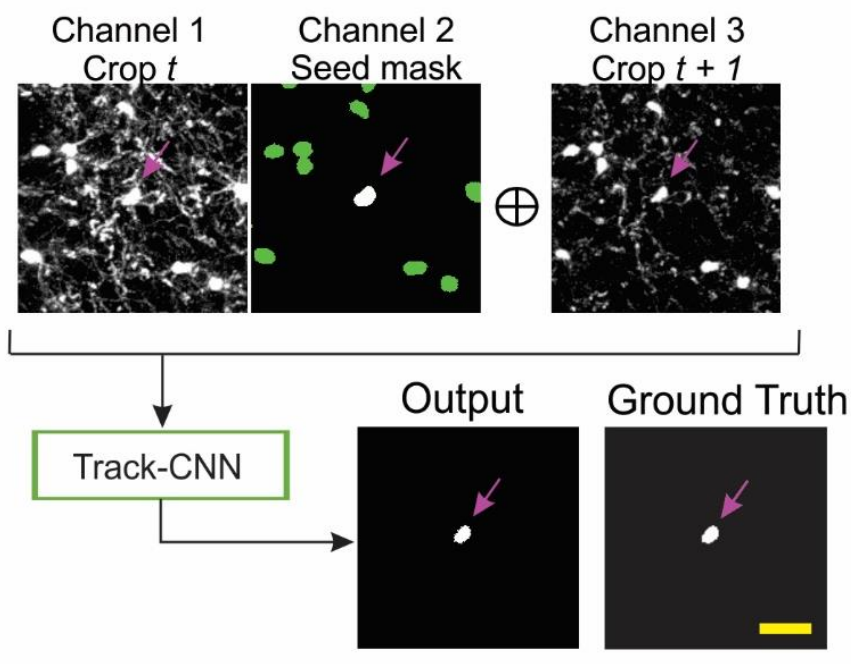

B
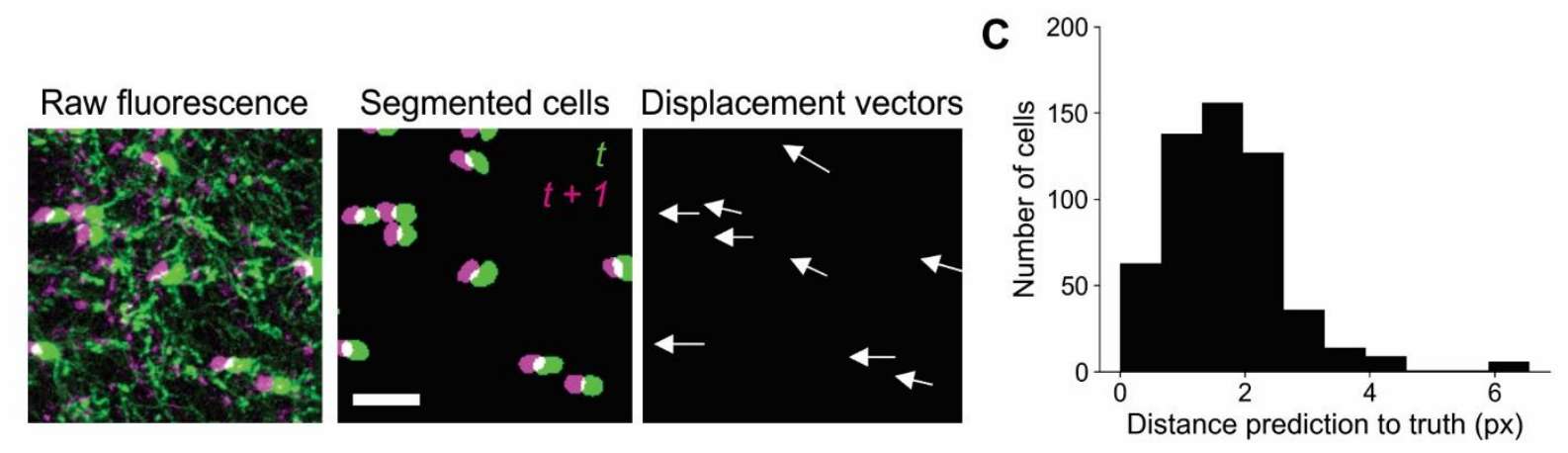

Figure 3: Track-CNN processing steps. (A) Crops are taken from each pair of timepoints $t$ and $t+1$ centered around a cell denoted by magenta arrow on channel 1 . Channel 1 contains raw fluorescence from timepoint $t$. Channel 2 contains seed mask of cell of interest (magenta arrow). Adjacent segmented cells are set to a lower value (green). Channel 3 contains raw fluorescence from timepoint $t+1$. Cropped images are concatenated together to form input to network. The network output is a semantic segmentation indicating the location of the seed masked cell on timepoint $t+1$. This procedure is repeated for all cells on all consecutive timepoints. Scale bar: $30 \mu \mathrm{m}$. (B) Example showing local coherence in how tracked cells in a local region shift between timepoint $t$ (green) and $t+1$ (magenta), allowing for predictive postprocessing using average movement vectors (right). Scale bar: $30 \mu \mathrm{m}$. (C) Distribution of distances from predicted to actual location of cell on timepoint $t+1$ given any cell on timepoint $t$. The prediction is generated by taking the average displacement vector of five nearest neighbor tracks. Differences between predicted and actual location were typically within 6 pixels. 
A

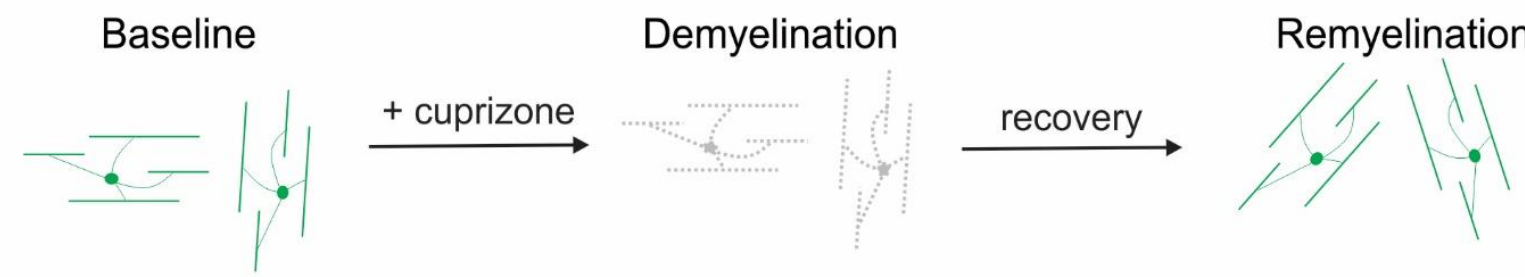

B

C
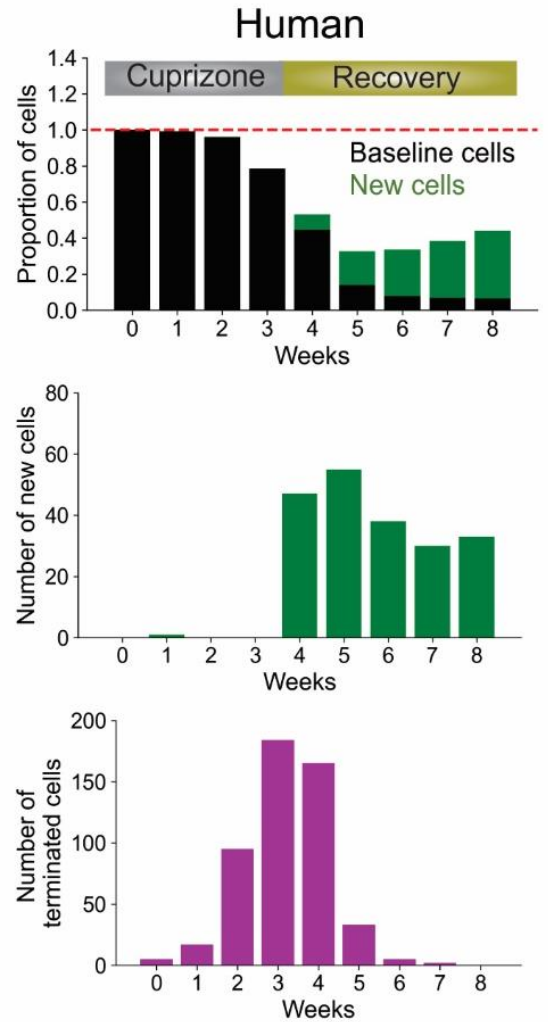

E

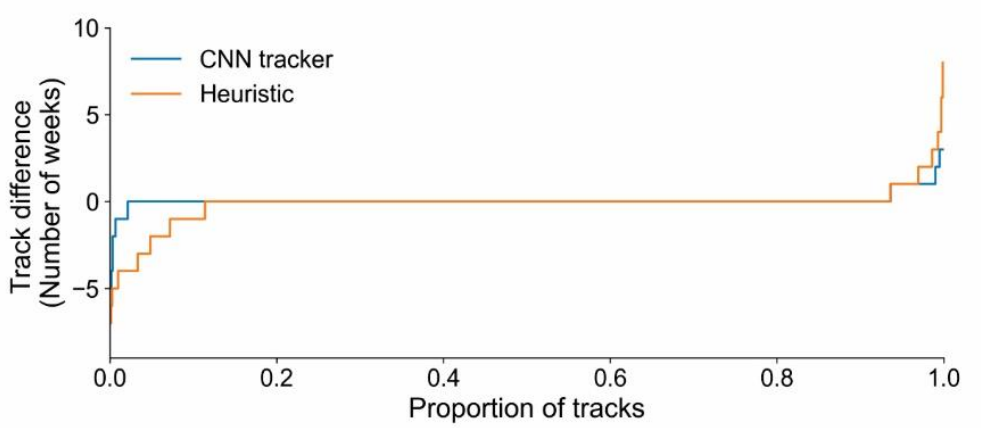

CNN
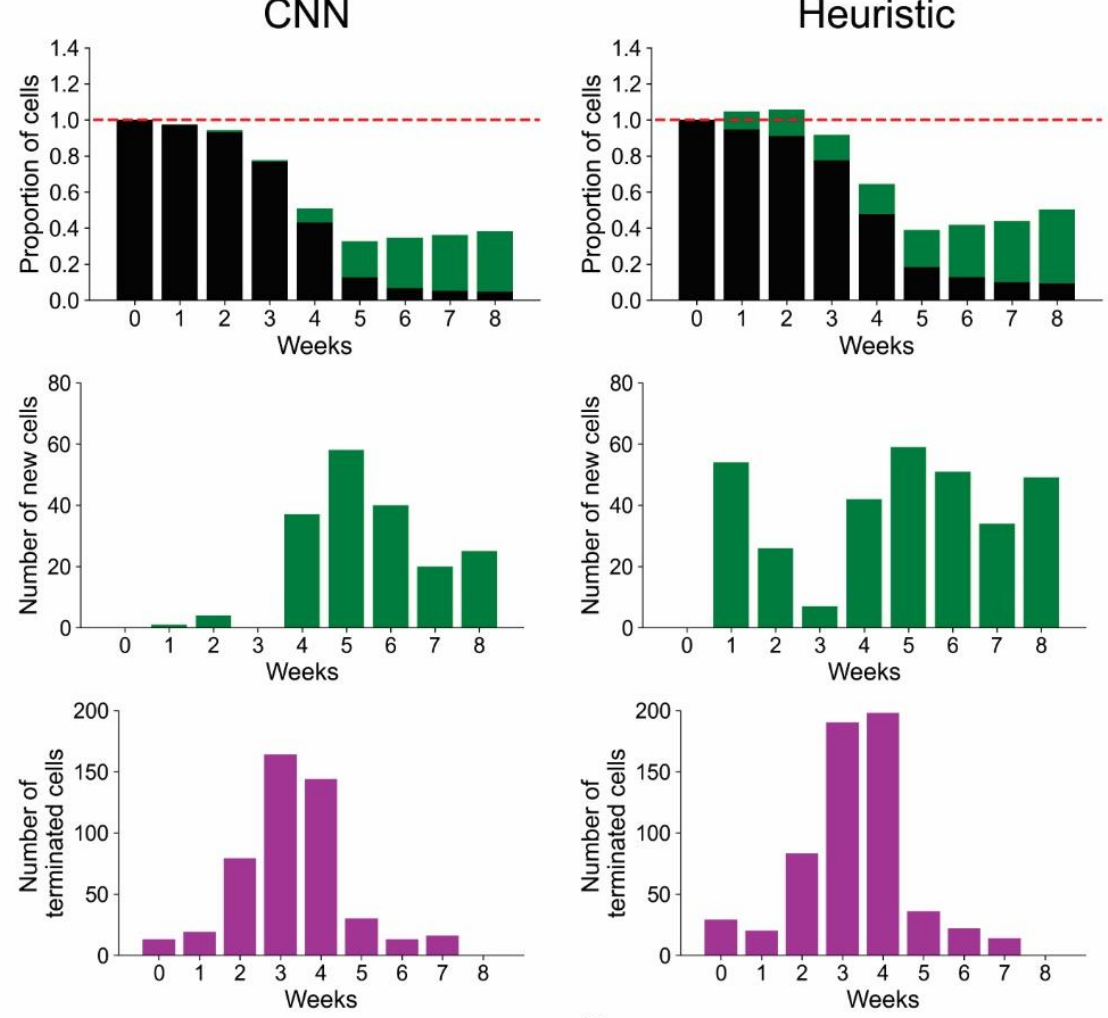

$\mathbf{F}$

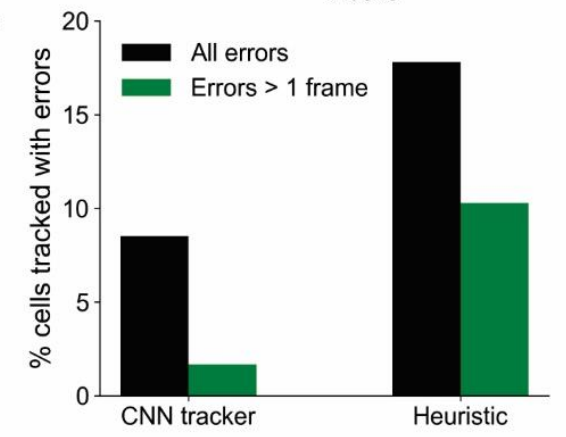

Figure 4: CNN-based tracking outperforms heuristic tracking. (A) Diagram illustrating cuprizone induced oligodendrocyte loss and recovery during the imaging period. (B) Overall normalized trends for human, CNN and heuristic tracking methods on test timeseries withheld from training data. (C) Number of new cells detected per timepoint for each method. (D) Number of cells terminated per timepoint for each method. (E) Track difference (length of track in ground truth - length of track by machine count) comparing ground truth to CNN and heuristic methods, respectively. (F) Comparison of major errors, defined as under- or over-tracking for $>1$ timepoint, and total errors by $\mathrm{CNN}$ and heuristic methods. 
A

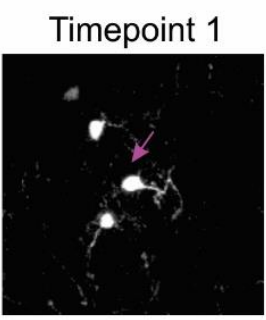

C
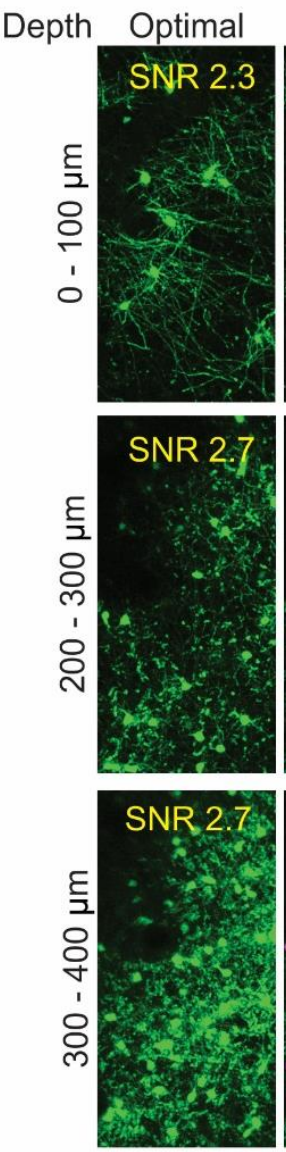
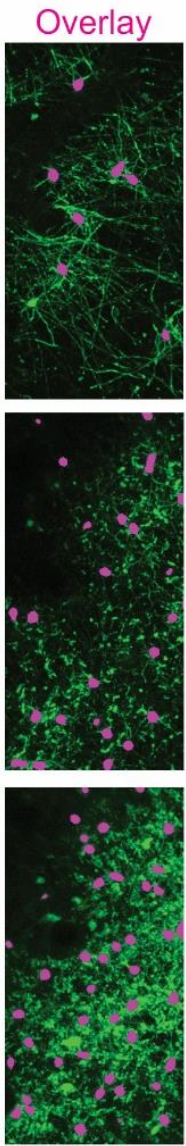

Timepoint 2

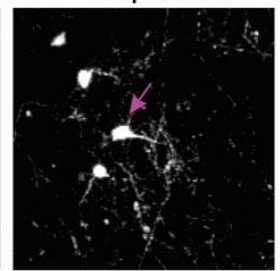

Ground Truth
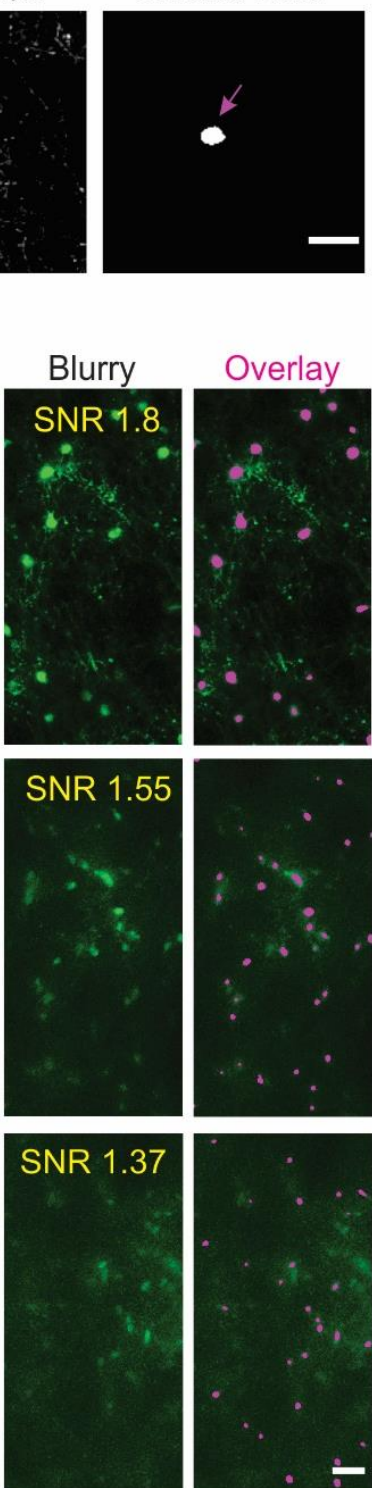
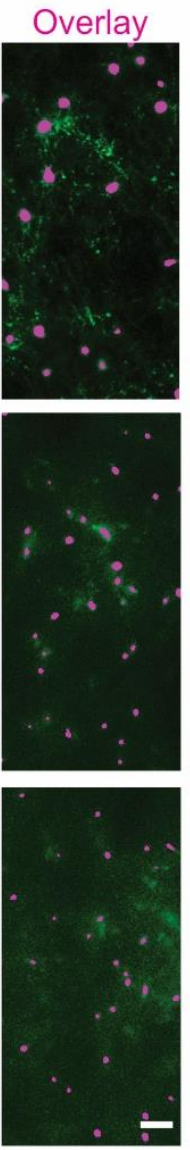

B
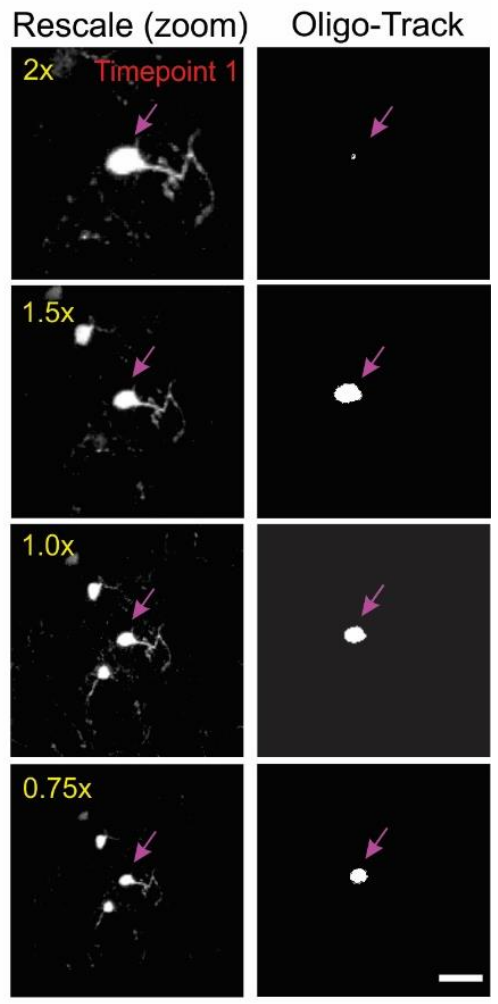

D

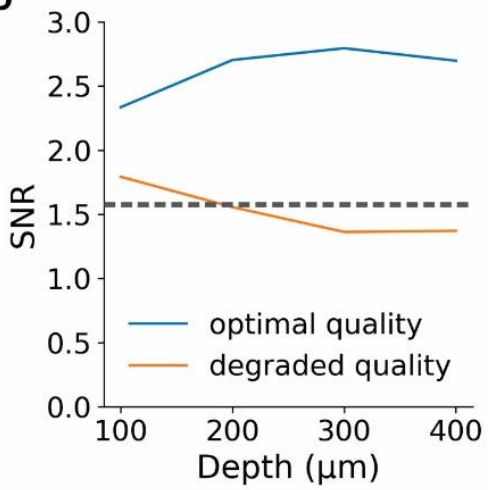

Figure 5: Oligo-Track enables robust cell tracking under different experimental on Track-CNN performance. Cell of interest denoted by magenta arrow. Scale bar: $30 \mu \mathrm{m}$. (C) Representative XY maximum projections at indicated SNR values and depths in a volume with optimal image quality (left), and a volume with a less transparent cranial window (right). Overlay of cells detected by Oligo-Track in Magenta. Scale bar: $30 \mu \mathrm{m}$. (D) Plotting average SNR across depth of optimal quality and degraded quality volumes. Dashed grey line indicates human perceptual limit for reliably tracing data. Also represents point at which algorithm will provide warning to user. 

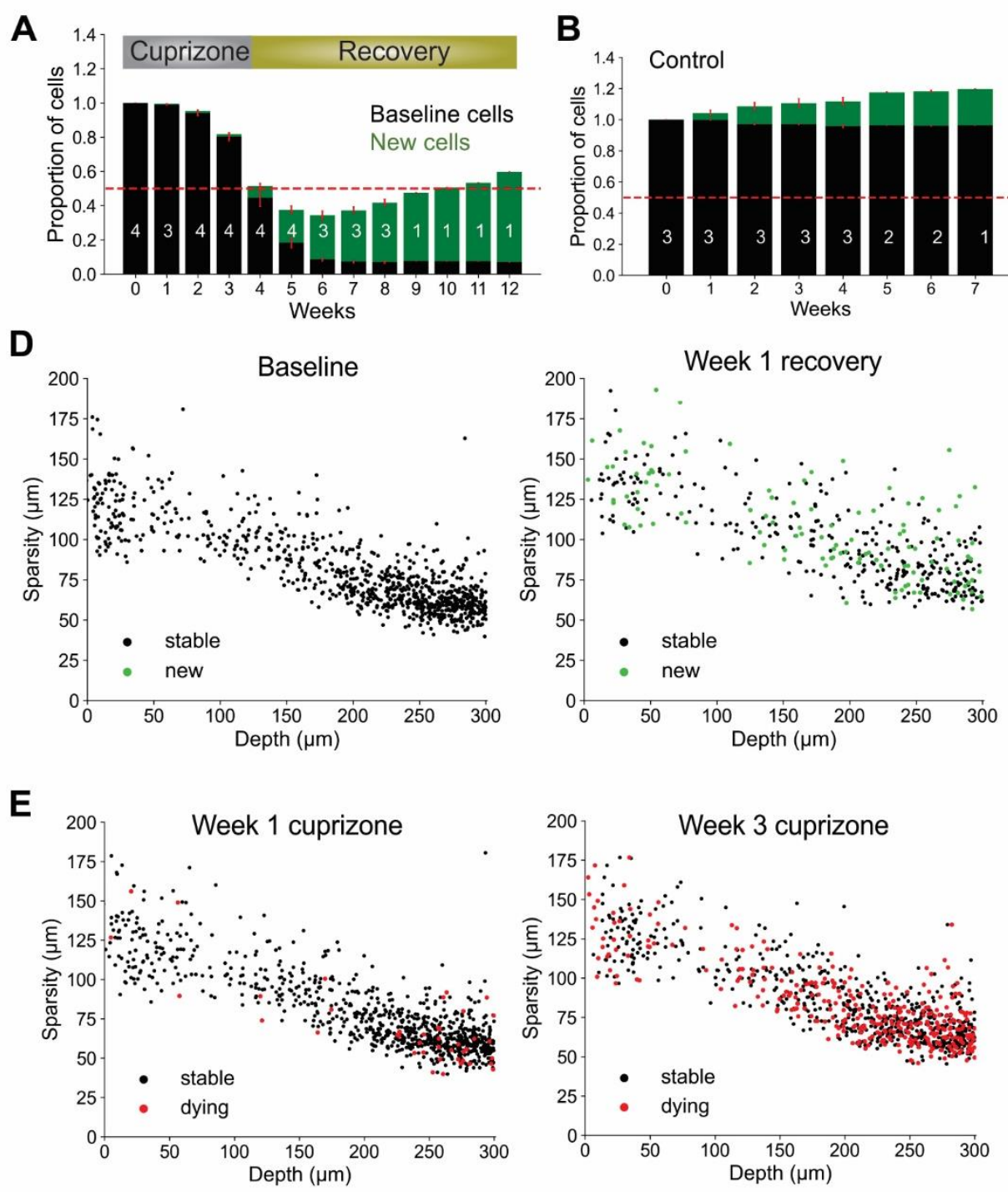

871

872

873

874

875

876

877

878

879

880

881

882

883

884

885

886

887

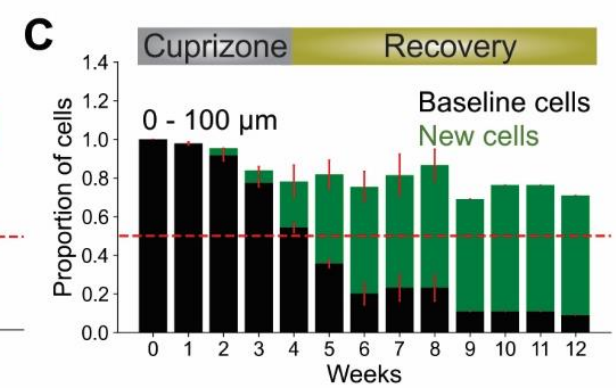

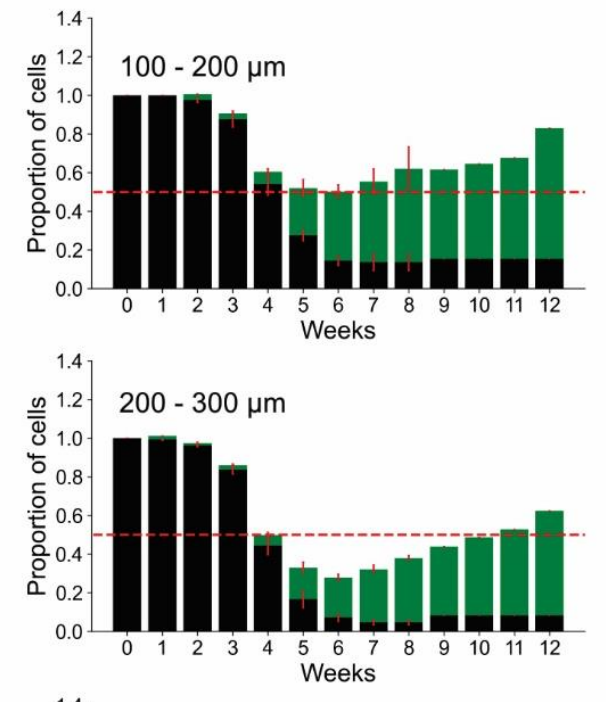
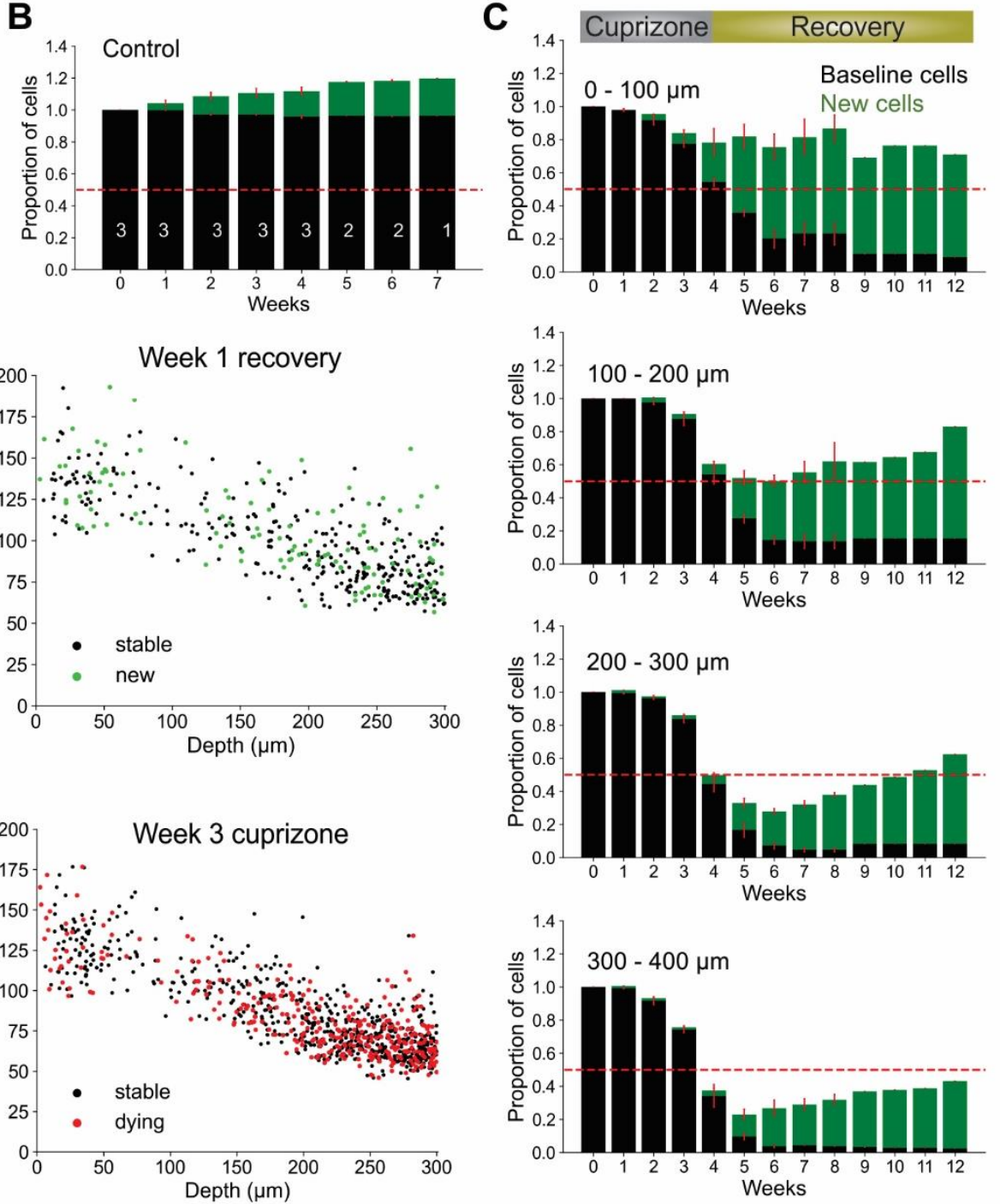

\section{Figure 6: Local oligodendrocyte density does not correlate with region specific} suppression of regeneration. (A) Normalized values for baseline and newly formed oligodendrocytes across weeks of cuprizone treatment and recovery. Bars indicate cell numbers averaged across animals for each week. (B) Normalized values for baseline and newly formed cells in no treatment condition. (C) Cortical depth-specific changes in oligodendrocyte regeneration showing suppressed regeneration in deeper layers. Volume split into 4 sections based on depth $(0-100 \mu \mathrm{m}, 100-200 \mu \mathrm{m}, 200-300 \mu \mathrm{m}, 300-400 \mu \mathrm{m})$. (D) Cell sparsity (average distance to 10 nearest neighbors) of stable and newly formed oligodendrocytes at baseline and week 2 of recovery shows no obvious clustering patterns at any timepoint. (E) Sparsity of cells that will die within one week (red) at week 1 and week 3 of cuprizone also shows no obvious clustering patterns at any timepoint. Cells pooled from $n=4$ cuprizone treated and $n=3$ control mice. 
A
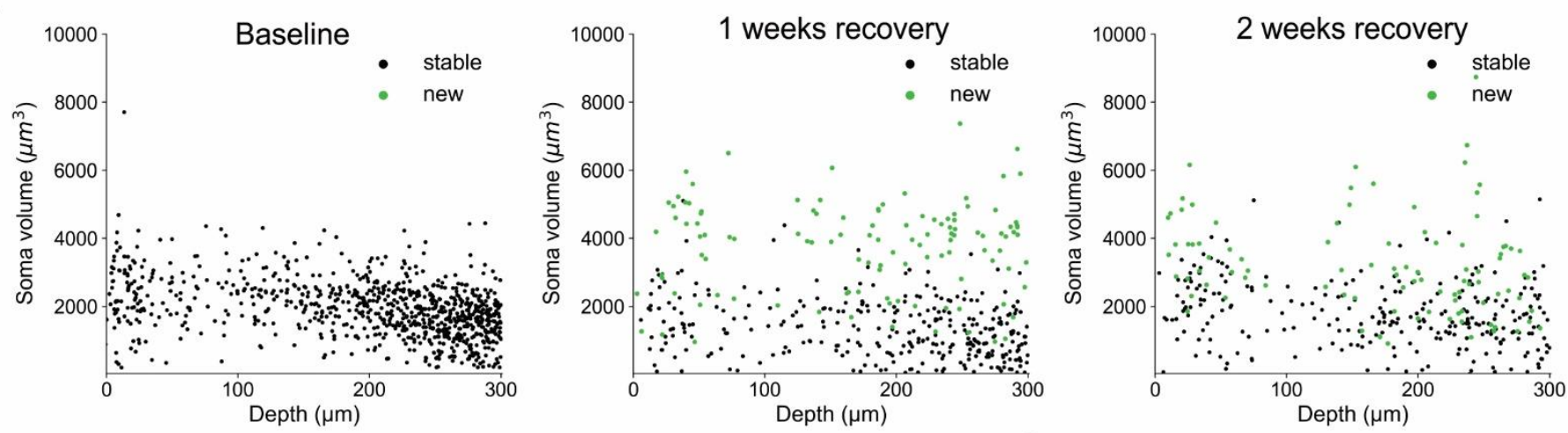

B
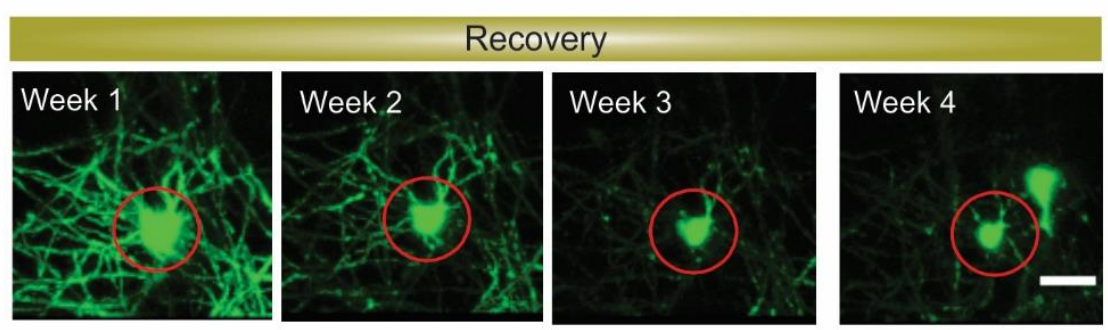

C

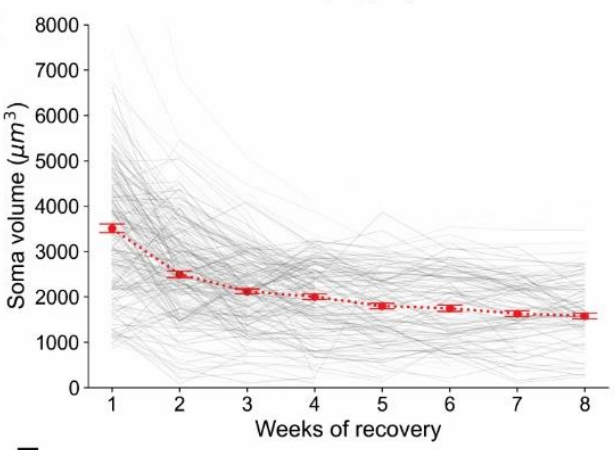

D

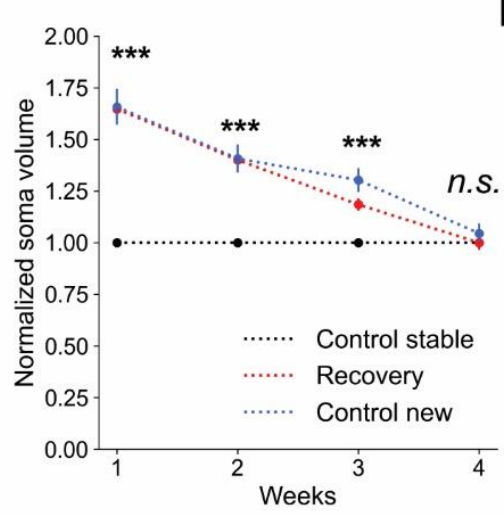

E

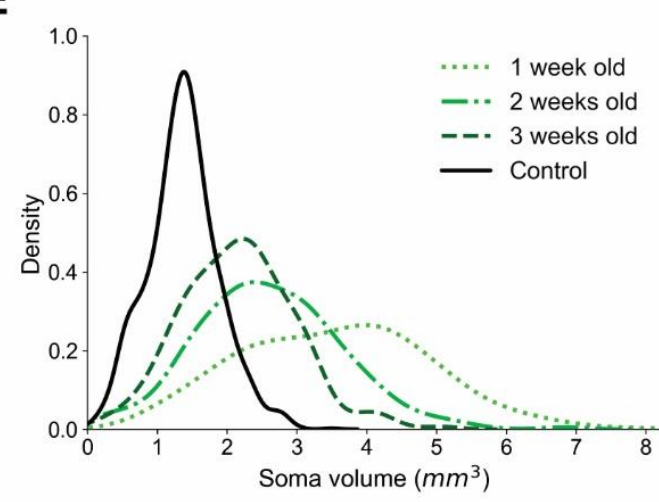

F

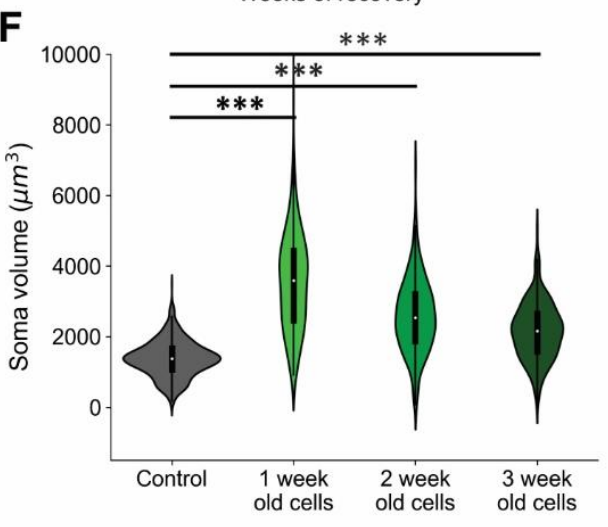

888

889

890

891

892

893

894

895

896

897

898

899

900

901

902

903

Figure 7: Newly generated oligodendrocytes can be identified by cell soma volume. (A) Soma volume of stable and newly formed oligodendrocytes at baseline, 1 week recovery, and 2 weeks recovery across different cortical depths. (B) Representative example of the change in soma size of newly formed oligodendrocyte tracked across 4 weeks of recovery. Scale bar: 20 $\mu \mathrm{m}$. (C) Plot of decrease in soma volume for 250 cells over weeks relative to time of cell generation. Red dots are mean \pm SEM. (D) Comparison of soma volume in newly formed oligodendrocytes during recovery compared with stable cells in non-treated mice. Also includes comparison of soma volume between spontaneously formed oligodendrocytes and stable cells in non-treated mice. All values are normalized to the mean soma volume of stable control cells at each matched timepoint. (E) Kernel density estimate for each distribution of soma volumes at indicated timepoints. These normalized distributions help visualize the probability that a cell with a certain soma volume is within a certain age range post-oligodendrogenesis. (F) Distribution of soma volumes of cells that are 1,2 , and 3 weeks old relative to cells in mice that are not treated with cuprizone at matched timepoints. Cells pooled from $n=4$ cuprizone treated and $n=3$ control mice. See Supplementary file 1 for statistical tests and significance level for each comparison. 
A
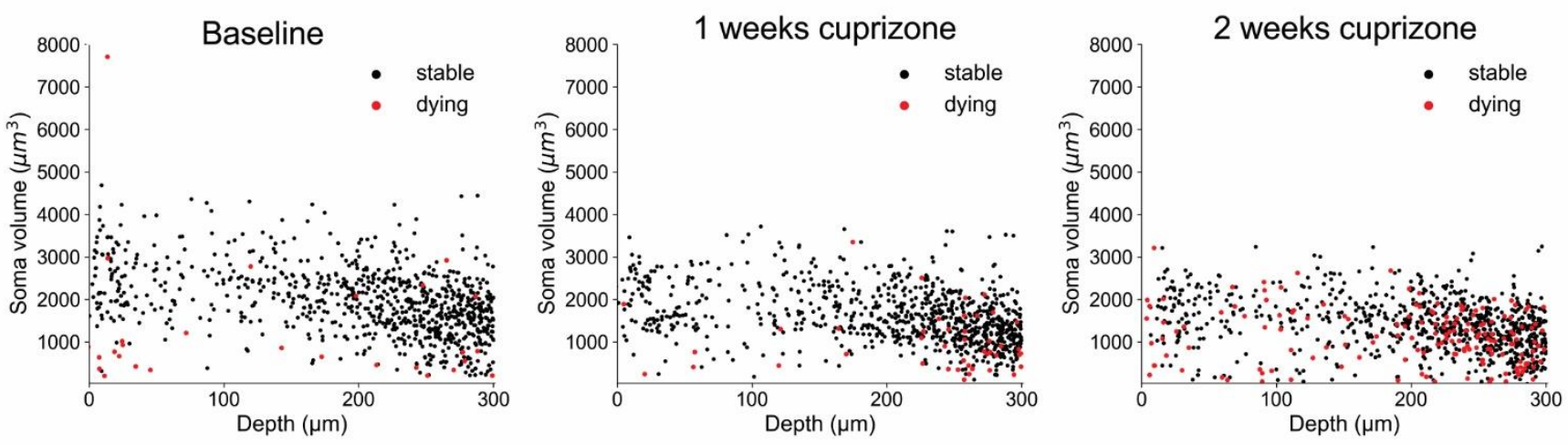

B
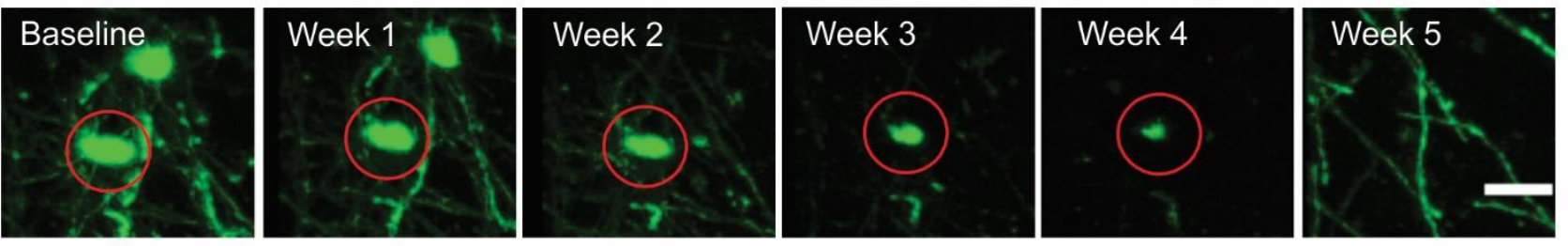

C

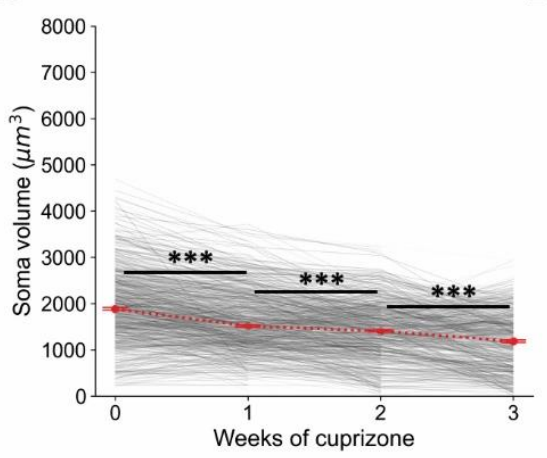

D

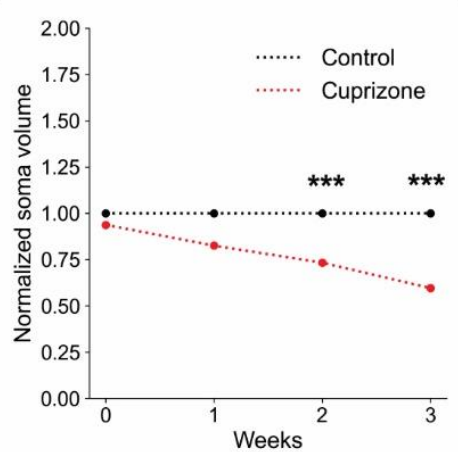

E

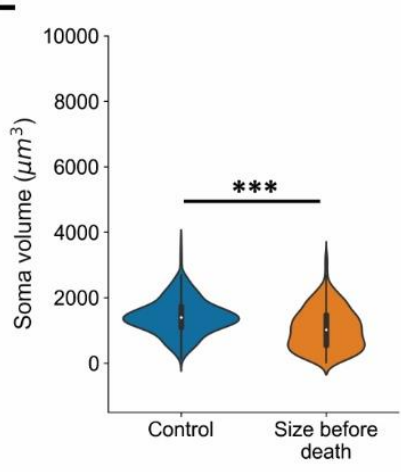

$\mathbf{F}$

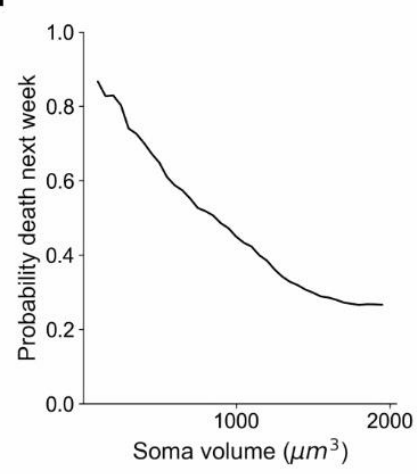

904

905

906

907

908

909

910

911

912

913

914

915

916

917

918

919

920

921

922
Figure 8: Oligodendrocyte death can be predicted from cell soma volume. (A) Volume of cell somata within 1 week of dying (red) at baseline, 1 week cuprizone, and 2 week cuprizone timepoints. (B) Representative example of cell soma shrinkage throughout cuprizone treatment, resulting in eventual death. Scale bar: $20 \mu \mathrm{m}$. (C) Plot of soma volume decrease for 860 cells during cuprizone treatment. (D) Plot of average soma volume of dying cells at each timepoint of cuprizone treatment relative to timepoint matched cells from control mice. All values are normalized to the mean soma volume of stable control cells at each matched timepoint. (E) Overall distribution of soma volumes for non-treated cells and cells within 1 week of death during cuprizone treatment. (F) Probability that a cell soma below a certain volume is within 1 week of death. Cells pooled from $n=4$ cuprizone treated and $n=3$ control mice. See Supplementary file 1 for statistical tests and significance level for each comparison.

Video 1: Cell tracking across two stable timepoints. Timepoint $t$ (left) and $t+1$ (right). Magenta indicates the cell that is currently undergoing assessment by Track-CNN. After assessment, a color is assigned to the cell on $t$ and $t+1$ to represent a tracked cell across timepoints. If the cell is untracked (or dies between timepoints), the cell soma is set to pure white on $t$. 
https://www.dropbox.com/s/nz9ll1n2ucw5v7w/Video 1 tracking stable cells compressed.mp4

924

925

926

927

928

929

930

931

932

933

934

935

936

?dl=0

Video 2: Cell tracking across cuprizone injury timepoints. Timepoint $t$ (left) and $t+1$ (right). Magenta indicates the cell that is currently undergoing assessment by Track-CNN. After assessment, a color is assigned to the cell on $t$ and $t+1$ to represent a tracked cell across timepoints. If the cell is untracked (or dies between timepoints), the cell soma is set to pure white on $t$.

\section{https://www.dropbox.com/s/zd647matnwsiokz/Video 2 tracking cuprizone cells compressed. $\underline{\mathrm{mp}} 4 ? \mathrm{dl}=0$}

Video 3: Cell sparsity over weeks of cuprizone treatment and recovery. Newly formed cells marked in green (left) and cells that will die within a week marked in red (right) starting from

https://www.dropbox.com/s/vaem5ancd2jz5fh/Video 3 sparsity over weeks compressed.avi? baseline followed by three weeks of cuprizone treatment and subsequent recovery.

941

942

943

944

945

946

947

948

$\underline{\mathrm{d} l=0}$

949

Video 4: Soma size of dying and newly formed cells over weeks of cuprizone treatment. Newly formed cells marked in green (left) and cells that will die within a week a marked in red (right) starting from baseline followed by three weeks of cuprizone treatment and subsequent recovery.

https://www.dropbox.com/s/vb2sgbilrcpuzdp/Video 4 volume over weeks compressed.avi?dl $=0$ 